Homology, Homotopy and Applications, vol.17(2), 2015, pp.317-341

\title{
LIN-WANG TYPE FORMULA FOR THE HAEFLIGER INVARIANT
}

\author{
KEIICHI SAKAI
}

\author{
(communicated by Dev P. Sinha)
}

\begin{abstract}
In this paper we study the Haefliger invariant for long embeddings $\mathbb{R}^{4 k-1} \hookrightarrow \mathbb{R}^{6 k}$ in terms of the self-intersections of their projections to $\mathbb{R}^{6 k-1}$, under the condition that the projection is a generic long immersion $\mathbb{R}^{4 k-1} \rightarrow \mathbb{R}^{6 k-1}$. We define the notion of "crossing changes" of the embeddings at the self-intersections and describe the change of the isotopy classes under crossing changes using the linking numbers of the double point sets in $\mathbb{R}^{4 k-1}$. This formula is a higher-dimensional analogue to that of X.-S. Lin and Z. Wang for the order 2 invariant for classical knots. As a consequence, we show that the Haefliger invariant is of order 2 in a similar sense to Birman and Lin. We also give an alternative proof for the result of M. Murai and K. Ohba concerning "unknotting numbers" of embeddings $\mathbb{R}^{3} \hookrightarrow \mathbb{R}^{6}$. Our formula enables us to define an invariant for generic long immersions $\mathbb{R}^{4 k-1} \rightarrow \mathbb{R}^{6 k-1}$ which are liftable to embeddings $\mathbb{R}^{4 k-1} \hookrightarrow \mathbb{R}^{6 k}$. This invariant corresponds to V. Arnold's plane curve invariant in Lin-Wang theory, but in general our invariant does not coincide with the order 1 invariant of T. Ekholm.
\end{abstract}

\section{Introduction}

A long $j$-embedding in $\mathbb{R}^{n}$ is an embedding $\mathbb{R}^{j} \hookrightarrow \mathbb{R}^{n}$ that is the standard inclusion outside a compact set. We denote by $\mathcal{K}_{n, j}$ the space of long $j$-embeddings in $\mathbb{R}^{n}$. Similarly, we denote the space of long immersions $\mathbb{R}^{j} \rightarrow \mathbb{R}^{n}$ by $\mathcal{I}_{n, j}$.

In [25] the author constructed, for some pairs $(n, j)$, a cochain map $I: \mathcal{D}^{*} \rightarrow$ $\Omega_{D R}^{*}\left(\mathcal{K}_{n, j}\right)$ from a complex $\mathcal{D}^{*}$ of graphs to the de Rham complex of $\mathcal{K}_{n, j}$ via configuration space integrals associated with graphs. For other interesting pairs - in particular, for $(n, j)=(6 k, 4 k-1)$ - the map $I$ has not yet been proved to be a cochain map, and it is not clear whether graph cocycles in $\mathcal{D}^{*}$ yield closed forms of $\mathcal{K}_{6 k, 4 k-1}$. But in [25] we found a cocycle $H \in \mathcal{D}^{*}$ and a differential form $c \in \Omega_{D R}^{0}\left(\mathcal{K}_{6 k, 4 k-1}\right)$ such that $\mathcal{H}:=I(H)+c \in \Omega_{D R}^{0}\left(\mathcal{K}_{6 k, 4 k-1}\right)$ is closed and is equal (up to sign) to the Haefliger invariant that gives a group isomorphism $\pi_{0}\left(\mathcal{K}_{6 k, 4 k-1}\right) \cong \mathbb{Z}$. This integral expression $\mathcal{H}$ looks very similar to that for the finite type invariant $v_{2}$ of order 2 (the Casson invariant) for classical knots $[\mathbf{4}, \mathbf{1 5}]$.

Received September 30, 2014, revised March 19 and June 3, 2015; published on December 3, 2015. 2010 Mathematics Subject Classification: 58D10, 81Q30, 57Q45, 57R40, 57R42.

Key words and phrases: the space of embeddings, the Haefliger invariant, configuration space integral, finite type invariant, generic immersion.

Article available at http://dx.doi.org/10.4310/HHA.2015.v17.n2.a15

Copyright (C) 2015, International Press. Permission to copy for private use granted. 
In this paper, based on the integral expression $\mathcal{H}$, we show that the Haefliger invariant indeed behaves similarly to $v_{2}$. To do this, we study $\mathcal{H}(f)$ under the condition that its projection $p \circ f \in \mathcal{I}_{6 k-1,4 k-1}$ is a generic immersion, where $p: \mathbb{R}^{6 k} \rightarrow$ $\mathbb{R}^{6 k-1}$ denotes the projection forgetting the last $6 k$-th coordinate. In contrast to the case of knots in $\mathbb{R}^{3}$, this is not a generic condition. But it is possible to move the generator of $\pi_{0}\left(\mathcal{K}_{6 k, 4 k-1}\right)$ by an isotopy so that the above condition is satisfied (see $\S 3$ ), and hence it is possible for all the embeddings. A generic immersion $g \in \mathcal{I}_{6 k-1,4 k-1}$ has only (possibly empty) transverse two-fold self-intersection $A=A_{1} \sqcup \cdots \sqcup A_{m} \subset \mathbb{R}^{6 k-1}$, where each $A_{i}$ is a connected, closed oriented $(2 k-1)$ dimensional manifold. If $g=p \circ f$ for some $f \in \mathcal{K}_{6 k, 4 k-1}$, then $g: g^{-1}\left(A_{i}\right) \rightarrow A_{i}$ is a trivial double covering and we write $g^{-1}\left(A_{i}\right)=L_{i}^{0}(f) \sqcup L_{i}^{1}(f) \subset \mathbb{R}^{4 k-1}$. We define the notion of the crossing changes at the "crossings" $A_{i}$, similarly as in the classical knot theory, and denote by $f_{S} \in \mathcal{K}_{6 k, 4 k-1}$ the embedding obtained from $f$ by crossing changes at $A_{i}, i \in S \subset\{1, \ldots, m\}$. In Theorem 2.5 we show that the difference $\mathcal{H}(f)-\mathcal{H}\left(f_{S}\right)$ can be described as a signed sum of the linking numbers $l k\left(L_{i}^{\epsilon}(f), L_{j}^{\epsilon^{\prime}}(f)\right)$ and $l k\left(L_{i}^{\epsilon}\left(f_{S}\right), L_{j}^{\epsilon^{\prime}}\left(f_{S}\right)\right), \epsilon, \epsilon^{\prime} \in\{0,1\}, i, j \in\{1, \ldots, m\}$. This formula is a higher-dimensional analogue to those for $v_{2}[\mathbf{1 6},(4.3)],[\mathbf{1 9},(3.2)],[\mathbf{2 0},(2.6)],[\mathbf{2 3}]$.

We define the notion of finite type invariants for $\mathcal{K}_{6 k, 4 k-1}$ in a similar manner to the Birman-Lin characterization [3], and as a corollary of Theorem 2.5 we see in Theorem 2.9 that the Haefliger invariant is of order 2. In this sense, the Haefliger invariant can be seen as a higher-dimensional analogue to $v_{2}$. It seems that, in some aspects, the geometric meaning of the Haefliger invariant is understood better (see, for example, $[\mathbf{1 4}, \mathbf{5}, \mathbf{1 7}, \mathbf{2 8}, \mathbf{2 9}]$ ) than that of finite type invariants for classical knots. Thus more detailed studies on the Haefliger invariant (and other cohomology classes in higher dimensions that can be described by some integrals) might shed light on the geometric meaning of finite type invariants. It might be possible that the notion of "finite type invariants" can also be characterized from the perspective of manifold calculus $[\mathbf{1 2}]$ (see also $[\mathbf{6 , 7}, \mathbf{1 8}, \mathbf{3 1}]$ ). See Remark 2.10 for some discussion. As another consequence, we reprove the result of Murai and Ohba [22] concerning the "unknotting numbers" of long embeddings $\mathbb{R}^{3} \hookrightarrow \mathbb{R}^{6}$.

Similarly to $v_{2}$, the invariant $\mathcal{H}$ is essentially the sum of two integrals $I(X), I(Y)$ over some configuration spaces, which correspond, respectively, to the graphs $X$ and $Y$ (see Figure 4.1). Our formula in Theorem 2.5 is obtained by clarifying the geometric meaning of $I(X)$ to some extent; we see in Proposition 5.3 that $I(X)$ is essentially a signed sum of the linking numbers of $L_{i}^{\epsilon}$ 's and can be thought of as a high-dimensional analogue to the Gauss diagram term in the formulas in $[\mathbf{1 6}, \mathbf{1 9}, \mathbf{2 0}, \mathbf{2 3}$. Using our formula, we can define an invariant $E$ of generic immersions $\mathbb{R}^{4 k-1} \uparrow \mathbb{R}^{6 k-1}$ that can be lifted to embeddings $\mathbb{R}^{4 k-1} \hookrightarrow \mathbb{R}^{6 k}$ (see Theorem 2.11). In fact, $E$ is the essential part of $I(Y)$ (see (6.1)). Our invariant $E$ is a high-dimensional analogue to Lin-Wang invariant $\alpha$ for generic plane curves [16, Definition 5.4]. The invariant $\alpha$ turns out to be a linear combination of the Arnold invariants for generic plane curves [1]. One might therefore expect that $E$ would be a linear combination of Ekholm's order 1 invariants $[\mathbf{1 0}, \mathbf{1 1}]$, which look analogous to the Arnold invariants. Contrary to the expectation, we see in Theorem 2.11 that in general $E$ is not of order 1 . In fact, we see that, when $k=1$, the jump of $E$ at self-tangency singularities can be computed using the linking numbers of links that arise as the double point set of immersion $\mathbb{R}^{3} \uparrow \mathbb{R}^{5}$, and the linking numbers can be arbitrarily large by the result of Ogasa [21]. 
This paper is organized as follows. In $\S 2$ we fix the notation and state the results. The main results are Theorems 2.5, 2.9 (proved in $\S 5$ ), and 2.11 (proved in $\S 6$ ). In $\S 3$ we show an explicit computation using Theorem 2.5. We review the graph complex $\mathcal{D}$ and our construction of $\mathcal{H}$ in $\S 4$.

\section{Acknowledgments}

The author expresses his deep appreciation to Masamichi Takase and Tadayuki Watanabe for their many fruitful suggestions. The author also feels grateful to Dev Sinha for the discussion about the finite type invariants. A question asked by Yuichi Yamada about $\mathcal{H}$ motivated the author to start this work. The author is partially supported by JSPS KAKENHI Grant number 25800038.

\section{Notation and results}

The self-intersection $A$ of an immersion $g: M \rightarrow N$ is $A:=\left\{q \in N|| g^{-1}(q) \mid \geqslant 2\right\}$, where $|S|$ is the cardinality of a set $S$. If $g \in \mathcal{I}_{6 k-1,4 k-1}$ is generic, then $\left|g^{-1}(q)\right|=2$ for any $q \in A$. Moreover, $A$ is a $(2 k-1)$-dimensional closed submanifold and $g: g^{-1}(A) \rightarrow$ $A$ is a double covering. We call $g^{-1}(A) \subset \mathbb{R}^{4 k-1}$ the double point set. Suppose that $g$ is liftable to $f \in \mathcal{K}_{6 k, 4 k-1}$-namely, $g=p \circ f$, where in general $p: \mathbb{R}^{n} \rightarrow \mathbb{R}^{n-1}$ is given by $p\left(x_{1}, \ldots, x_{n}\right)=\left(x_{1}, \ldots, x_{n-1}\right)$ - then $g: g^{-1}(A) \rightarrow A$ is a trivial double covering. Let $A_{i} \subset \mathbb{R}^{6 k-1}(i=1,2, \ldots)$ be path components of $A$, and we call each $A_{i}$ a crossing of (the "knot diagram" $p \circ f$ of) $f$. We set $g^{-1}\left(A_{i}\right)=L_{i}^{0} \sqcup L_{i}^{1}=L_{i}^{0}(f) \sqcup L_{i}^{1}(f)$. Each $L_{i}^{\epsilon} \subset \mathbb{R}^{4 k-1}$ is a $(2 k-1)$-dimensional connected closed submanifold. By convention, $f\left(L_{i}^{1}\right) \subset \mathbb{R}^{6 k}$ sits "above" $f\left(L_{i}^{0}\right)$-namely, if $\boldsymbol{x}^{\epsilon}=\left(x_{1}, \ldots, x_{6 k-1}, x_{6 k}^{\epsilon}\right) \in f\left(L_{i}^{\epsilon}\right), \epsilon=$ $0,1\left(\right.$ so $\left.p\left(\boldsymbol{x}^{0}\right)=p\left(\boldsymbol{x}^{1}\right)\right)$, then $x_{6 k}^{0}<x_{6 k}^{1}$.

Remark 2.1. Any $f \in \mathcal{K}_{6 k, 4 k-1}$ can be moved by an isotopy so that $p \circ f$ is a generic immersion; indeed such an isotopy exists for the embedding $\mathcal{S}$ which generates $\pi_{0}\left(\mathcal{K}_{6 k, 4 k-1}\right)$ (see $\S 3$ ). But the condition for $f$ that $p \circ f$ is a generic immersion is not generic, and in general such an isotopy is not "small."

Remark 2.2. Not all $g \in \mathcal{I}_{6 k-1,4 k-1}$ are regularly homotopic to any liftable immersion, in contrast to the case of plane curves. Indeed, as shown in $[\mathbf{3 0}, \S 3], g \in \mathcal{I}_{5,3}$ is regularly homotopic to a liftable immersion if and only if its Smale invariant $\pi_{0}\left(\mathcal{I}_{5,3}\right) \stackrel{\cong}{\rightarrow} \mathbb{Z}$ is even.

Lemma 2.3 ([10, Lemma 5.1.3], [11, Proposition 3.3]). For any $f \in \mathcal{K}_{6 k, 4 k-1}$ as above, the submanifolds $A_{i} \subset \mathbb{R}^{6 k}$ and $L_{i}^{\epsilon} \subset \mathbb{R}^{4 k-1}$ admit natural orientations.

Proof. Given a basis $\vec{u}=\left(\boldsymbol{u}_{1}, \ldots, \boldsymbol{u}_{2 k-1}\right)$ of $T_{x} A_{i}\left(x \in A_{i}\right)$, we can choose tangent frames $\vec{v}=\left(\boldsymbol{v}_{2 k}, \ldots, \boldsymbol{v}_{4 k-1}\right)$ and $\vec{w}=\left(\boldsymbol{w}_{2 k}, \ldots, \boldsymbol{w}_{4 k-1}\right)$ of the two sheets of $p \circ f$ meeting at $x \in A_{i}$ so that $(\vec{u}, \vec{v})$ and $(\vec{u}, \vec{w})$ are the positive bases of these two sheets. We say $\vec{u}$ represents the positive orientation of $A_{i}$ if $(\vec{u}, \vec{v}, \vec{w})$ is a positive basis of $\mathbb{R}^{6 k-1}$. Since the codimension of $p \circ f$ is even, this definition is independent of the order of the two sheets. We orient $L_{i}^{\epsilon}, \epsilon=0,1$, so that $p \circ f: L_{i}^{\epsilon} \rightarrow A_{i}$ preserves the orientation. 
To simplify the computations, we often move $f \in \mathcal{K}_{6 k, 4 k-1}$ to a special position.

Definition 2.4 (see Figure 2.1). We say an embedding $f \in \mathcal{K}_{6 k, 4 k-1}$ is almost planar if

(i) the composite $p \circ f: \mathbb{R}^{4 k-1} \leftrightarrow \rightarrow \mathbb{R}^{6 k-1}$ is a generic immersion,

(ii) $f\left(\mathbb{R}^{4 k-1}\right) \subset \mathbb{R}^{6 k-1} \times[0, \delta]$ for a small $\delta>0$, and

(iii) $f\left(\mathbb{R}^{4 k-1} \backslash \bigcup_{i} N\left(L_{i}^{1}\right)\right) \subset \mathbb{R}^{6 k-1} \times\{0\}$, where $N\left(L_{i}^{\epsilon}\right) \subset \mathbb{R}^{4 k-1}$ are closed tubular neighborhoods of $L_{i}^{\epsilon}$ in $\mathbb{R}^{4 k-1}$ such that $N\left(L_{i}^{\epsilon}\right) \cap N\left(L_{j}^{\epsilon^{\prime}}\right)=\emptyset$ if $(i, \epsilon) \neq\left(j, \epsilon^{\prime}\right)$.

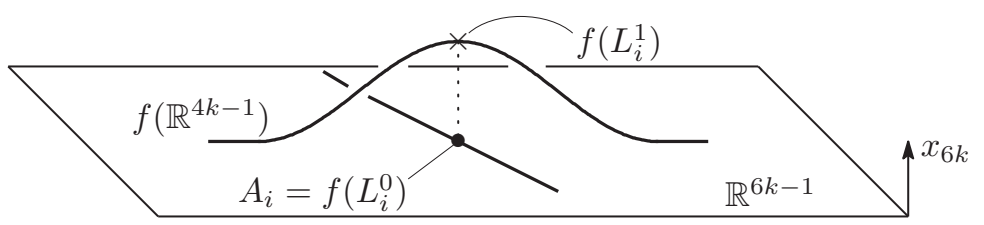

Figure 2.1: An almost planar embedding

If $f \in \mathcal{K}_{6 k, 4 k-1}$ is such that $p \circ f \in \mathcal{I}_{6 k-1,4 k-1}$ is generic, then we can transform $f$ to be almost planar without changing $p \circ f$, by an isotopy in the $x_{6 k}$-direction. Notice that if $f$ is almost planar, then the crossings $A_{i}$ are equal to $f\left(L_{i}^{0}\right)$.

Suppose that $f$ is almost planar and that the self-intersection of $p \circ f$ has $m$ components. For $S \subset\{1, \ldots, m\}$, let $f_{S} \in \mathcal{K}_{6 k, 4 k-1}$ be defined by

$$
f_{S}(x):= \begin{cases}\iota(f(x)) & x \in N\left(L_{i}^{1}\right), i \in S, \\ f(x) & \text { otherwise, }\end{cases}
$$

where $\iota: \mathbb{R}^{6 k} \rightarrow \mathbb{R}^{6 k}$ is given by $\iota\left(x_{1}, \ldots, x_{6 k}\right)=\left(x_{1}, \ldots, x_{6 k-1},-x_{6 k}\right)$. We say $f_{S}$ is an embedding obtained from $f$ by crossing changes at the crossings $\left\{A_{i}\right\}_{i \in S}$. Notice that $p \circ f=p \circ f_{S}$ and

$$
L_{i}^{\epsilon}\left(f_{S}\right)= \begin{cases}L_{i}^{\epsilon+1}(f) & i \in S, \\ L_{i}^{\epsilon}(f) & i \notin S ;\end{cases}
$$

here $\epsilon$ is understood to be in $\mathbb{Z} / 2=\{0,1\}$ and $1+1=0$.

Let $\mathcal{H}: \mathcal{K}_{6 k, 4 k-1} \rightarrow \mathbb{Z}$ be the Haefliger invariant (see $\S 4$ for our construction). Our main theorem describes the difference $\mathcal{H}(f)-\mathcal{H}\left(f_{S}\right)$ using the linking numbers of $L_{i}^{\epsilon}$ 's.

Theorem 2.5. Let $f \in \mathcal{K}_{6 k, 4 k-1}$ be such that $p \circ f$ is a generic immersion and has the nonempty self-intersection $A=A_{1} \sqcup \cdots \sqcup A_{m}$. Then for any $S \subset\{1, \ldots, m\}$,

$$
\begin{aligned}
& \mathcal{H}(f)-\mathcal{H}\left(f_{S}\right) \\
& =\frac{1}{4}\left(\sum_{(i, \epsilon)<\left(j, \epsilon^{\prime}\right)}(-1)^{\epsilon+\epsilon^{\prime}} l k\left(L_{i}^{\epsilon}(f), L_{j}^{\epsilon^{\prime}}(f)\right)-\sum_{(i, \epsilon)<\left(j, \epsilon^{\prime}\right)}(-1)^{\epsilon+\epsilon^{\prime}} l k\left(L_{i}^{\epsilon}\left(f_{S}\right), L_{j}^{\epsilon^{\prime}}\left(f_{S}\right)\right)\right) \\
& =\frac{1}{2} \sum_{\begin{array}{c}
(i, \epsilon)<\left(j, \epsilon^{\prime}\right), \\
(\text { exactly one of } i, j) \in S
\end{array}}(-1)^{\epsilon+\epsilon^{\prime}} l k\left(L_{i}^{\epsilon}(f), L_{j}^{\epsilon^{\prime}}(f)\right),
\end{aligned}
$$


where lk stands for the linking number, and we write $(i, \epsilon)<\left(j, \epsilon^{\prime}\right)$ if $i<j$ or if $i=j$, $\epsilon=0, \epsilon^{\prime}=1$.

(2.2) follows from (2.1); if $i, j \notin S$, then $l k\left(L_{i}^{\epsilon}(f), L_{j}^{\epsilon^{\prime}}(f)\right)=l k\left(L_{i}^{\epsilon}\left(f_{S}\right), L_{j}^{\epsilon^{\prime}}\left(f_{S}\right)\right)$ is contained in both sums in (2.1) with the same sign $(-1)^{\epsilon+\epsilon^{\prime}}$ and cancels out. If $i, j \in S$, then $l k\left(L_{i}^{\epsilon}(f), L_{j}^{\epsilon^{\prime}}(f)\right)=l k\left(L_{i}^{\epsilon+1}\left(f_{S}\right), L_{j}^{\epsilon^{\prime}+1}\left(f_{S}\right)\right)$ is contained in both sums with the same sign $(-1)^{\epsilon+\epsilon^{\prime}}$ and cancels out. If $i \in S$ and $j \notin S$, then $l k\left(L_{i}^{\epsilon}(f), L_{j}^{\epsilon^{\prime}}(f)\right)$ is contained in the first sum with sign $(-1)^{\epsilon+\epsilon^{\prime}}$ while $l k\left(L_{i}^{\epsilon}(f), L_{j}^{\epsilon^{\prime}}(f)\right)=l k\left(L_{i}^{\epsilon+1}\left(f_{S}\right), L_{j}^{\epsilon^{\prime}}\left(f_{S}\right)\right)$ is contained in the second sum with the opposite sign $(-1)^{\epsilon+1+\epsilon^{\prime}}$.

Remark 2.6. The formula in Theorem 2.5 is similar to Lin and Wang's formula for the Casson invariant $v_{2}[\mathbf{1 6}]$; given a diagram of $f \in \mathcal{K}_{3,1}$, let $f_{S} \in \mathcal{K}_{3,1}$ be obtained by changing the crossings $c_{i}, i \in S$. Then a slight generalization of [16, (4.3)] can be written as

$$
v_{2}(f)-v_{2}\left(f_{S}\right)=\frac{1}{4}\langle\curvearrowleft \curvearrowleft, G(f)\rangle-\frac{1}{4}\left\langle\curvearrowleft \curvearrowleft, G\left(f_{S}\right)\right\rangle,
$$

where $G(f)$ is the Gauss diagram of (the diagram of) $f$ and $\langle\ldots \ldots, G(f)\rangle$ is the sum of $\varepsilon_{1} \varepsilon_{2}$ for all the subdiagrams of $G(f)$ of the shape $\stackrel{\varepsilon_{1}}{\varepsilon_{2}}\left(\varepsilon_{i}= \pm 1\right.$ are the signs of the corresponding crossings of the diagrams). This kind of pairing appears elsewhere, for example, in [23]. Choosing an $S$ that yields a "descending diagram" $f_{S}$ (and hence $f_{S}$ is trivial), and computing the right-hand side of (2.3), we reprove the Polyak-Viro formula [23, Theorem 1.A]. Regarding the pairing as the sum of the linking numbers of "0-dimensional Hopf links $S^{0} \sqcup S^{0} \hookrightarrow \mathbb{R}^{1}$ " determined by the crossings of the diagrams, we can say that Theorem 2.5 is a higher-dimensional analogue to (2.3) and to the Polyak-Viro formula.

Theorem 2.5 together with the result of Ogasa [21] gives an alternative proof for the following result of Murai and Ohba [22], which states that the "unknotting number" of any nontrivial embedding $f \in \mathcal{K}_{6,3}$ is 1 (see $\S 6.4$ for the proof).

Corollary $2.7([\mathbf{2 2}])$. Any nontrivial $f \in \mathcal{K}_{6,3}$ can be unknotted by a crossing change at a single crossing. Namely, $f$ is isotopic to some $f^{\prime}$, with $f_{\{1\}}^{\prime}$ isotopic to the trivial inclusion.

The proof is outlined as follows: Given any two-component link $L \subset \mathbb{R}^{3}$, by a result of Ogasa $[\mathbf{2 1}]$ we can find $f_{0}: \mathbb{R}^{3} \hookrightarrow \mathbb{R}^{6}$ such that it is isotopic to the trivial inclusion and its projection $p \circ f: \mathbb{R}^{3} \rightarrow \mathbb{R}^{5}$ has $L$ as its double point set. By Theorem 2.5 the embedding $f_{1}$ obtained by the crossing change along $L$ satisfies $\mathcal{H}\left(f_{1}\right)=-l k(L)$. This means that any $f \in \mathcal{K}_{6,3}$ with arbitrary $\mathcal{H}(f)$ can be obtained by a single crossing change from $f_{0}$. See $\S 6.4$ for details. In $[\mathbf{2 2}]$ an explicit way to unknot the generator of $\pi_{0}\left(\mathcal{K}_{6,3}\right)$ (and its connected sums) by a single crossing change is given.

Below we introduce the notion of finite type invariants for $\mathcal{K}_{6 k, 4 k-1}$. As a consequence of Theorem 2.5, we prove in $\S 5$ that $\mathcal{H}$ is an invariant of order 2. 
Definition 2.8. Let $u: \mathcal{K}_{6 k, 4 k-1} \rightarrow \mathbb{R}$ be a function, and let $A=\left\{A_{i}\right\}_{1 \leqslant i \leqslant s+1}$ be a (sub)set of crossings of $p \circ f$, where $f \in \mathcal{K}_{6 k, 4 k-1}$ is almost planar. Define

$$
V_{s+1}(u)(f):=\sum_{S \subset\{1, \ldots, s+1\}}(-1)^{|S|} u\left(f_{S}\right) .
$$

An isotopy invariant $u$ is said to be of order $s$ if $V_{s+1}(u)=0$.

Theorem 2.9. The Haefliger invariant is of order 2.

Remark 2.10. Our Definition 2.8 of "finite type invariants" is modeled after [3] and is similar to those in $[\mathbf{1 3}, \mathbf{3 3}]$. Finite type invariants can also in some cases be characterized as the functions that factor through stages of the Taylor tower [12]. Volić uses Bott-Taubes integrals to prove in $[\mathbf{3 2}$, Theorem 4.5$]$ that $\mathbb{R}$-valued invariants of order $k$ factor through the $2 k$-th stage of the homology Taylor tower for $\mathcal{K}_{3,1}$. In [6] it is proved that $v_{2}: \mathcal{K}_{3,1} \rightarrow \mathbb{Z}$ factors through the third stage of the homotopy Taylor tower for $\mathcal{K}_{3,1}$. Budney, Conant, Koytcheff, and Sinha [7] show that the $(k+1)$-st stage of the homotopy tower defines order $k$ invariants, and based on spectral sequence calculations conjecture any additive invariant of order $k$ factors through this tower.

In general, the equivalence between these two characterizations of finiteness, Birman-Lin and Goodwillie-Weiss, is not known. Theorem 2.9 together with a result of Munson [18] proves the equivalence for $\mathcal{K}_{6 k, 4 k-1}$.

In $\S 6$ we define an invariant for generic liftable immersion $\mathbb{R}^{4 k-1} \rightarrow \mathbb{R}^{6 k-1}$ and discuss some of its properties.

Theorem 2.11. Let $g \in \mathcal{I}_{6 k-1,4 k-1}$ be a liftable generic immersion-namely, $g=$ $p \circ f$ for some $f \in \mathcal{K}_{6 k, 4 k-1}$. Choose a lift $f \in \mathcal{K}_{6 k, 4 k-1}$ and define

$$
E(g):=\mathcal{H}(f)-\frac{1}{4} \sum_{(i, \epsilon)<\left(j, \epsilon^{\prime}\right)}(-1)^{\epsilon+\epsilon^{\prime}} l k\left(L_{i}^{\epsilon}, L_{j}^{\epsilon^{\prime}}\right) .
$$

Then $E$ is independent of the choice of $f$ and is invariant of generic immersions. $E$ varies at the strata of non-generic immersions as described in Lemmas 6.4, 6.5, and 6.7. In the case $k=1$, the invariant $E$ is not of order 1 in the sense of Ekholm $[10,11]$.

$E$ is an invariant of generic immersions because the linking numbers are constant unless the isotopy class of the self-intersection of $p \circ f$ changes. That $E$ is well defined follows from our formula in Theorem 2.5. See $\S 6.1$.

Remark 2.12. The invariant $E$ is a high-dimensional analogue to the Lin-Wang invariant $\alpha$ [16, Definition 5.4] for generic plane curves; let $g \in \mathcal{I}_{2,1}$ be a generic plane curve, and let $f \in \mathcal{K}_{3,1}$ be its lift-namely, $g=p \circ f$. Then (2.3) implies that

$$
\alpha(g):=v_{2}(f)-\frac{1}{4}\langle\curvearrowright \curvearrowleft, G(f)\rangle
$$

is independent of the choice $f$. The invariant $\alpha$ is in fact equal to a linear combination of the Arnold invariants $J^{ \pm}$and $S t$ of a plane curve [1]. On the other hand, Ekholm $[10, \S 6.1],[11, \S 4]$ defined invariants for generic immersions $M^{n m-1} \rightarrow N^{n(m+1)-1}$ that behave similarly to the Arnold invariants (for us, $m=2, n=2 k$ ). One may thus expect that $E$ might be a linear combination of Ekholm invariants, but Theorem 2.11 says that in general it is not the case. 


\section{Example}

Using Theorem 2.5, we show $\mathcal{H}(\mathcal{S})= \pm 1$ for Haefliger's generator $\mathcal{S}$ of $\pi_{0}\left(\mathcal{K}_{6 k, 4 k-1}\right) \cong \mathbb{Z}[\mathbf{1 4}]$. Fix $\alpha, \beta>0$ so that $2 \beta<\alpha$. Consider the Borromean ring $X \sqcup Y \sqcup Z \subset \mathbb{R}^{6 k}$, where

$$
\begin{aligned}
& X:=\partial\left\{(\mathbf{0}, \boldsymbol{y}, \boldsymbol{z}) \in\left(\mathbb{R}^{2 k}\right)^{\times 3}|| \boldsymbol{y}|\leqslant \alpha,| \boldsymbol{z} \mid \leqslant \beta\right\} \approx S^{4 k-1}, \\
& Y:=\partial\left\{(\boldsymbol{x}, \mathbf{0}, \boldsymbol{z}) \in\left(\mathbb{R}^{2 k}\right)^{\times 3}|| \boldsymbol{z}|\leqslant \alpha,| \boldsymbol{x} \mid \leqslant \beta\right\} \approx S^{4 k-1}, \\
& Z:=\partial\left\{(\boldsymbol{x}, \boldsymbol{y}, \mathbf{0}) \in\left(\mathbb{R}^{2 k}\right)^{\times 3}|| \boldsymbol{x}|\leqslant \alpha,| \boldsymbol{y} \mid \leqslant \beta\right\} \approx S^{4 k-1}
\end{aligned}
$$

(see Figure 3.1), and smooth their corners to get smooth $(4 k-1)$-spheres (denoted by $X, Y, Z$ again). $\mathcal{S}$ is defined as the connected-sum $\mathcal{S}:=X \sharp Y \sharp Z \sharp f_{0}$, where $f_{0}: \mathbb{R}^{4 k-1} \subset$ $\mathbb{R}^{6 k}$ is (isotopic to) the standard inclusion.
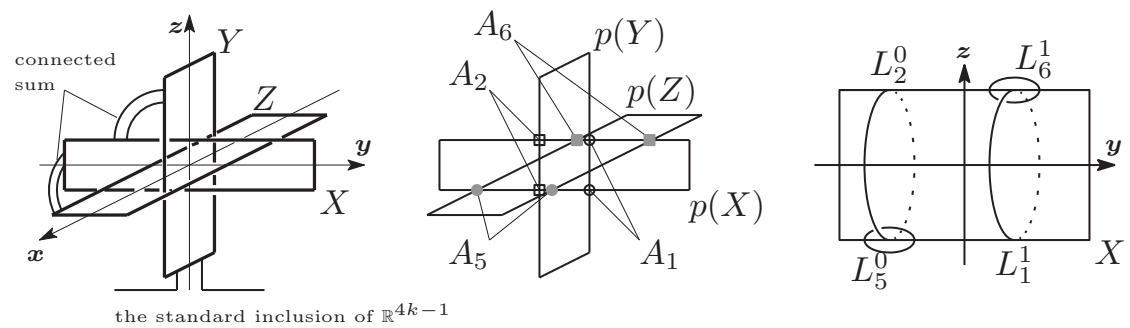

Figure 3.1: Haefliger's generator $\mathcal{S}$ and the self-intersection of $p \circ \mathcal{S}$

Let $\boldsymbol{n}:=(1, \ldots, 1) \in \mathbb{R}^{6 k}$, and consider the projection $p: \mathbb{R}^{6 k} \rightarrow(\mathbb{R} \boldsymbol{n})^{\perp}$, instead of $\mathbb{R}^{6 k} \rightarrow \mathbb{R}^{6 k-1} \times\{0\}$. Then $p \circ \mathcal{S}$ is generic, as seen in Figure 3.1. To detect $p(X) \cap p(Y)$, find $(\mathbf{0}, \boldsymbol{y}, \boldsymbol{z}) \in X$ and $t \in \mathbb{R}$ satisfying $(\mathbf{0}, \boldsymbol{y}, \boldsymbol{z})+t \boldsymbol{n} \in Y$. In fact, $p(X) \cap p(Y)=A_{1} \sqcup A_{2}$ has two components, and the double point set $L_{i}^{\epsilon} \subset \mathbb{R}^{4 k-1}$ satisfying $A_{i}=p\left(\mathcal{S}\left(L_{i}^{0}\right)\right)=p\left(\mathcal{S}\left(L_{i}^{1}\right)\right) \quad(i=1,2) \quad$ is given as follows: put $\quad \boldsymbol{n}^{\prime}:=$ $(1, \ldots, 1) \in \mathbb{R}^{2 k}$ and $\beta^{\prime}:=\beta / \sqrt{2 k}$; then

$$
\begin{array}{ll}
L_{1}^{0}=\left\{\left(-\beta^{\prime} \boldsymbol{n}^{\prime}, \mathbf{0}, \boldsymbol{z}\right) \in Y|| \boldsymbol{z}+\beta^{\prime} \boldsymbol{n}^{\prime} \mid=\beta\right\}, & L_{1}^{1}=\left\{\left(\mathbf{0}, \beta^{\prime} \boldsymbol{n}^{\prime}, \boldsymbol{z}\right) \in X|| \boldsymbol{z} \mid=\beta\right\}, \\
L_{2}^{0}=\left\{\left(\mathbf{0},-\beta^{\prime} \boldsymbol{n}^{\prime}, \boldsymbol{z}\right) \in X|| \boldsymbol{z} \mid=\beta\right\}, & L_{2}^{1}=\left\{\left(\beta^{\prime} \boldsymbol{n}^{\prime}, \mathbf{0}, \boldsymbol{z}\right) \in Y|| \boldsymbol{z}-\beta^{\prime} \boldsymbol{n}^{\prime} \mid=\beta\right\}
\end{array}
$$

(in the computation we use $2 \beta<\alpha$ ). By symmetry we see that $p(Y) \cap p(Z)=A_{3} \sqcup A_{4}$ has two components, and the double point sets satisfying $A_{i}=p\left(\mathcal{S}\left(L_{i}^{0}\right)\right)=p\left(\mathcal{S}\left(L_{i}^{1}\right)\right)$ $(i=3,4)$ are given as

$$
\begin{array}{ll}
L_{3}^{0}=\left\{\left(\boldsymbol{x},-\beta^{\prime} \boldsymbol{n}^{\prime}, \mathbf{0}\right) \in Z|| \boldsymbol{x}+\beta^{\prime} \boldsymbol{n}^{\prime} \mid=\beta\right\}, & L_{3}^{1}=\left\{\left(\boldsymbol{x}, \mathbf{0}, \beta^{\prime} \boldsymbol{n}^{\prime}\right) \in Y|| \boldsymbol{x} \mid=\beta\right\}, \\
L_{4}^{0}=\left\{\left(\boldsymbol{x}, \mathbf{0},-\beta^{\prime} \boldsymbol{n}^{\prime}\right) \in Y|| \boldsymbol{x} \mid=\beta\right\}, & L_{4}^{1}=\left\{\left(\boldsymbol{x}, \beta^{\prime} \boldsymbol{n}^{\prime}, \mathbf{0}\right) \in Z|| \boldsymbol{x}-\beta^{\prime} \boldsymbol{n}^{\prime} \mid=\beta\right\} .
\end{array}
$$

Similarly, $p(Z) \cap p(X)=A_{5} \sqcup A_{6}$ satisfies $A_{i}=p\left(\mathcal{S}\left(L_{i}^{0}\right)\right)=p\left(\mathcal{S}\left(L_{i}^{1}\right)\right)(i=5,6)$, where $L_{5}^{0}=\left\{\left(\mathbf{0}, \boldsymbol{y},-\beta^{\prime} \boldsymbol{n}^{\prime}\right) \in X|| \boldsymbol{y}+\beta^{\prime} \boldsymbol{n}^{\prime} \mid=\beta\right\}, L_{5}^{1}=\left\{\left(\beta^{\prime} \boldsymbol{n}^{\prime}, \boldsymbol{y}, \mathbf{0}\right) \in Z|| \boldsymbol{y} \mid=\beta\right\}$, $L_{6}^{0}=\left\{\left(-\beta^{\prime} \boldsymbol{n}^{\prime}, \boldsymbol{y}, \mathbf{0}\right) \in Z|| \boldsymbol{y} \mid=\beta\right\}, \quad L_{6}^{1}=\left\{\left(\mathbf{0}, \boldsymbol{y}, \beta^{\prime} \boldsymbol{n}^{\prime}\right) \in X|| \boldsymbol{y}-\beta^{\prime} \boldsymbol{n}^{\prime} \mid=\beta\right\}$.

If we take the connected-sum in the construction of $\mathcal{S}$ suitably, then there are no self-intersections of $p \circ \mathcal{S}$ other than $A_{1} \sqcup \cdots \sqcup A_{6}$. The corner smoothing does not cause any trouble; for example, any $\left(\mathbf{0}, \boldsymbol{y},-\beta^{\prime} \boldsymbol{n}^{\prime}\right) \in L_{5}^{0}$ satisfies $|\boldsymbol{y}| \leqslant 2 \beta<\alpha$ and $L_{5}^{0}$ 
does not touch the corner. All $L_{i}^{\epsilon}$ are $S^{2 k-1}$ and they form six disjoint Hopf links

$$
L_{1}^{1} \sqcup L_{6}^{1}, L_{2}^{0} \sqcup L_{5}^{0} \subset X, \quad L_{1}^{0} \sqcup L_{4}^{0}, L_{2}^{1} \sqcup L_{3}^{1} \subset Y, \quad L_{3}^{0} \sqcup L_{6}^{0}, L_{4}^{1} \sqcup L_{5}^{1} \subset Z,
$$

all of whose linking numbers are by symmetry equal to each other. Since we can unknot $\mathcal{S}$ by the crossing change at $A_{1}$, by $(2.2)$

$$
\mathcal{H}(\mathcal{S})=\frac{1}{2}\left((-1)^{1+1} l k\left(L_{1}^{1}, L_{6}^{1}\right)+(-1)^{0+0} l k\left(L_{1}^{0}, L_{4}^{0}\right)\right)= \pm \frac{1}{2}(1+1)= \pm 1 .
$$

\section{A review of an integral expression of the Haefliger invariant}

\subsection{Graph complex and configuration space integral}

Here we briefly recall the cochain complex $\mathcal{D}^{*}=\mathcal{D}_{n, j}^{*}$ of oriented graphs and the linear map $I: \mathcal{D}^{*} \rightarrow \Omega_{D R}^{*}\left(\mathcal{K}_{n, j}\right)$ that the author defined in [25] generalizing those in $[\mathbf{8}, \mathbf{9}, \mathbf{3 3}]$. The map $I$ is a cochain map under some conditions on $n, j$ and graphs. See $[\mathbf{2 5}, \mathbf{2 6}]$ for details.

By a graph we mean a graph with two types of vertices (called $i$ - and e-vertices) and two types of edges (called $\eta$ - and $\theta$-edges). The sets of i- and e-vertices, $\eta$ - and $\theta$-edges of a graph $\Gamma$ are denoted by, respectively, $V_{\mathrm{i}}(\Gamma), V_{\mathrm{e}}(\Gamma), E_{\eta}(\Gamma)$, and $E_{\theta}(\Gamma)$. We give the weights $j, n, j-1$, and $n-1$ to the elements of $V_{\mathrm{i}}(\Gamma), V_{\mathrm{e}}(\Gamma), E_{\eta}(\Gamma)$, and $E_{\theta}(\Gamma)$. An orientation of a graph is a choice of ordering of the weighted set $V_{\mathrm{i}}(\Gamma) \sqcup V_{\mathrm{e}}(\Gamma) \sqcup E_{\eta}(\Gamma) \sqcup E_{\theta}(\Gamma)$ together with the orientations of edges, modulo even permutations. Reversing $\eta$ - and $\theta$-edges changes the sign of orientation of graphs by $(-1)^{j}$ and $(-1)^{n}$, respectively. Figure 4.1 shows examples of graphs; i- and e-vertices are depicted by $\bullet$ and $\circ$, respectively, and $\eta$ - and $\theta$-edges are depicted by solid and dotted arrows, respectively (Figure 4.1 shows graphs for even $n$ and the orientation of the $\theta$-edges are omitted). The numbers assigned to vertices and edges indicate the ordering. Denote by $\mathcal{D}^{p, q}$ the vector space spanned by oriented graphs of order $p$ and
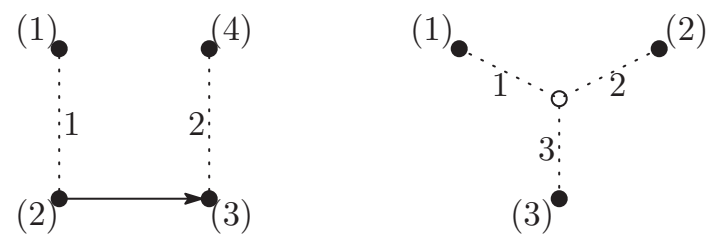

Figure 4.1: Graphs $X$ and $Y$

of degree $q$, where

$$
\operatorname{ord}(\Gamma):=\left|E_{\theta}(\Gamma)\right|-\left|E_{\mathrm{e}}(\Gamma)\right|, \quad \operatorname{deg}(\Gamma):=2\left|E_{\theta}(\Gamma)\right|-3\left|E_{\mathrm{e}}(\Gamma)\right|-\left|E_{\mathrm{i}}(\Gamma)\right| .
$$

For an oriented graph $\Gamma$ consider the configuration space

$$
C_{\Gamma}^{\circ}:=\left\{(f ; \boldsymbol{x} ; \boldsymbol{y}) \in \mathcal{K}_{n, j} \times \operatorname{Conf}_{\left|V_{\mathrm{i}}(\Gamma)\right|}^{\circ}\left(\mathbb{R}^{j}\right) \times \operatorname{Conf}_{\left|V_{\mathrm{e}}(\Gamma)\right|}^{\circ}\left(\mathbb{R}^{n}\right) \mid f\left(x_{i}\right) \neq y_{j}(\forall i, j)\right\},
$$

where $\operatorname{Conf}_{m}^{\circ}(M):=M^{\times m} \backslash \bigcup_{i<j}\left\{x_{i} \neq x_{j}\right\}$ stands for the usual configuration space. 
To each $\eta$-edge $\overrightarrow{i j}$ and $\theta$-edge $\overrightarrow{s t}$, we assign the generalized Gauss maps

$$
\begin{aligned}
& \varphi_{i j}^{\eta}: C_{\Gamma}^{\circ} \rightarrow S^{j-1}, \quad(f ; \boldsymbol{x} ; \boldsymbol{y}) \mapsto \frac{\boldsymbol{x}_{j}-\boldsymbol{x}_{i}}{\left|\boldsymbol{x}_{j}-\boldsymbol{x}_{i}\right|}, \\
& \varphi_{s t}^{\theta}: C_{\Gamma}^{\circ} \rightarrow S^{n-1}, \quad(f ; \boldsymbol{x} ; \boldsymbol{y}) \mapsto \frac{\boldsymbol{z}_{t}-\boldsymbol{z}_{s}}{\left|\boldsymbol{z}_{t}-\boldsymbol{z}_{s}\right|},
\end{aligned}
$$

where

$$
z_{s}:= \begin{cases}f\left(x_{s}\right) & \text { if } s \text { is an i-vertex } \\ y_{s} & \text { if } s \text { is an e-vertex. }\end{cases}
$$

Choose a representative of the orientation of $\Gamma$ so that $\theta$-edges follow after $\eta$-edges. Let $\varphi_{\Gamma}: C_{\Gamma}^{\circ} \rightarrow\left(S^{j-1}\right)^{\times\left|E_{\eta}(\Gamma)\right|} \times\left(S^{n-1}\right)^{\times\left|E_{\theta}(\Gamma)\right|}$ be the product of all these Gauss maps assigned to the edges of $\Gamma$. The product is taken in the order of the orientation of $\Gamma$. Let $\operatorname{vol}_{S^{N-1}} \in \Omega_{D R}^{N-1}\left(S^{N-1}\right)(N=n, j)$ be a unit volume form of $S^{N-1}$ that is (anti-)invariant under the action of $O(N)$ fixing the poles $\left\{ \pm e_{N}\right\} \subset S^{N-1}$, where $e_{N}:=(0, \ldots, 0,1) \in \mathbb{R}^{N}$. Define $\omega_{\Gamma} \in \Omega_{D R}^{*}\left(C_{\Gamma}^{\circ}\right)$ by

$$
\omega_{\Gamma}:=\varphi_{\Gamma}^{*}\left(\operatorname{vol}_{S^{j-1}}^{\times\left|E_{\eta}(\Gamma)\right|} \times \operatorname{vol}_{S^{j-1}}^{\times\left|E_{\theta}(\Gamma)\right|}\right),
$$

where $\operatorname{vol}_{S^{a-1}} \times \operatorname{vol}_{S^{b-1}}$ is the product of volume forms pulled back on $S^{a-1} \times S^{b-1}$ by the projections. Integrating $\omega_{\Gamma}$ along the fibers of the natural projections

$$
\pi_{\Gamma}: C_{\Gamma}^{\circ} \rightarrow \mathcal{K}_{n, j}
$$

(whose fibers are subspaces of usual configuration spaces), we obtain

$$
I(\Gamma):=\pi_{\Gamma * \omega_{\Gamma}} \in \Omega_{D R}^{*}\left(\mathcal{K}_{n, j}\right) .
$$

$I(\Gamma)$ is independent of the representative of the orientation of $\Gamma$. The degree of $I(\Gamma)$ can be given using $\operatorname{ord}(\Gamma), \operatorname{deg}(\Gamma)$, and the first betti number of $\Gamma$ (thought of as a 1-dimensional cell complex); see [25, §3].

Remark 4.1. The above integrals converge since we may replace $C_{\Gamma}^{\circ}$ with its (fiberwise) Fulton-MacPherson compactification (see [27]) denoted by $C_{\Gamma}$, over which the generalized Gauss maps are smoothly extended. Thus we obtain a linear map $I: \mathcal{D}^{*} \rightarrow \Omega_{D R}^{*}\left(\mathcal{K}_{n, j}\right)$.

By the generalized Stokes' theorem, $d I(\Gamma)$ is a linear combination of integrals along the codimension 1 boundary faces of the fibers of $\pi_{\Gamma}$, which are subspaces of compactified configuration spaces (Remark 4.1). The boundary faces of the compactifications are stratified according to the "complexity of collisions of points." The strata in which exactly two points collide are called principal. The author proved in [25] that, if the volume forms are (anti-)invariant, many such integrals along the non-principal boundary faces cancel out or vanish; the large part of the proof follows the arguments in $[\mathbf{8}, \mathbf{9}, \mathbf{3 3}]$. Thus if we define the coboundary maps $\delta: \mathcal{D}^{p, q} \rightarrow \mathcal{D}^{p, q+1}$ as the signed sum of graphs obtained by collapsing two vertices together with the edges between them (see $[\mathbf{2 5}, \S 2.3]$ for signs), the map $I$ becomes a cochain map. More precisely, the following holds.

Theorem 4.2 ([25, Theorem 1.2]). The map I is a cochain map if

- restricted to the subcomplex of tree graphs and $n, j$ are of same parity, or 
- restricted to the subcomplex of graphs of betti number not greater than 1 and both $n, j$ are odd.

Conjecturally, the map $I$ would always be a cochain map and a quasi-isomorphism. In fact, $\mathcal{D}^{*}$ looks very similar to the graph complexes given in $[\mathbf{2}]$ that compute the rational homology and homotopy of $\mathcal{K}_{n, j}$ in the stable dimensions. The map $I$ yields many non-zero cohomology classes even in the non-stable dimensions and in some dimensions not necessarily satisfying the condition in Theorem 4.2; for example, $H_{D R}^{3}\left(\mathcal{K}_{5,2}\right) \neq 0$. See $[\mathbf{2 6}, \mathbf{3 3}]$.

\subsection{The Haefliger invariant}

Let $X, Y \in \mathcal{D}^{2,0}$ be graphs in Figure 4.1, and denote $C_{4,0}:=C_{X}$ and $C_{3,1}:=C_{Y}$. We note that $C_{4,0}=\mathcal{K}_{6 k, 4 k-1} \times \operatorname{Conf}_{4}^{\circ}\left(\mathbb{R}^{4 k-1}\right)$ and $C_{3,1} \subset \mathcal{K}_{6 k, 4 k-1} \times \operatorname{Conf}_{3}^{\circ}\left(\mathbb{R}^{4 k-1}\right) \times$ $\mathbb{R}^{6 k}$. In fact, $H:=X / 2+Y / 6 \in \mathcal{D}^{2,0}$ is a cocycle and $I(H)$ is a 0 -form of $\mathcal{K}_{6 k, 4 k-1}$. Unfortunately, Theorem 4.2 might fail for $(n, j)=(6 k, 4 k-1)$; at present, it is not known whether the integral along the "anomalous boundary face" $\Sigma_{3,1} \subset \partial C_{3,1}$ (where all the four points $f\left(\boldsymbol{x}_{1}\right), f\left(\boldsymbol{x}_{2}\right), f\left(\boldsymbol{x}_{3}\right)$, and $\boldsymbol{x}_{4}$ collapse to a single point) vanishes or not. In [25] we add a correction term $c$ (defined below) to $I(H)$ to kill the anomalous contribution and get a closed-form $\mathcal{H}:=I(H)+c$.

The correction term $c$ is defined as follows. The interior Int $\Sigma_{3,1}$ of $\Sigma_{3,1}$ can be described by the following pullback square:

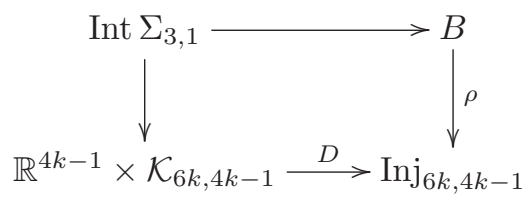

Let us explain the spaces and maps in the above diagram. $\operatorname{Inj}_{6 k, 4 k-1}$ is the space of linear, injective maps $\mathbb{R}^{4 k-1} \hookrightarrow \mathbb{R}^{6 k}$. The space $B$ is defined as

$$
\begin{aligned}
B:=\left\{\left(\lambda ;\left(\boldsymbol{x}_{1}, \boldsymbol{x}_{2}, \boldsymbol{x}_{3}\right) ; \boldsymbol{x}_{4}\right) \in \operatorname{Inj}_{6 k, 4 k-1} \times \operatorname{Conf}_{3}^{\circ}\left(\mathbb{R}^{4 k-1}\right) \times \mathbb{R}^{6 k} \mid\right. \\
\left.\lambda\left(\boldsymbol{x}_{i}\right) \neq \boldsymbol{x}_{4}, 1 \leqslant i \leqslant 3\right\} / \mathbb{R}_{+}^{1} \ltimes \mathbb{R}^{4 k-1},
\end{aligned}
$$

where $\mathbb{R}_{+}^{1} \ltimes \mathbb{R}^{4 k-1}$ acts diagonally on $\operatorname{Conf}_{3}^{\circ}\left(\mathbb{R}^{4 k-1}\right) \times \mathbb{R}^{6 k}$ as the positive scalings and translations along $\lambda\left(\mathbb{R}^{4 k-1}\right)$. The map $\rho$ is the natural projection, and $D$ is the differential

$$
D(x ; f):=\left(d f_{x}: T_{x} \mathbb{R}^{4 k-1}=\mathbb{R}^{4 k-1} \hookrightarrow \mathbb{R}^{6 k}=T_{f(x)} \mathbb{R}^{6 k}\right) .
$$

For $i=1,2,3$, the map

$$
\psi_{i}: B \rightarrow S^{6 k-1}, \quad\left[\iota ;\left(\boldsymbol{x}_{1}, \boldsymbol{x}_{2}, \boldsymbol{x}_{3}\right) ; \boldsymbol{x}_{4}\right] \mapsto \frac{\boldsymbol{x}_{4}-\iota\left(\boldsymbol{x}_{i}\right)}{\left|\boldsymbol{x}_{4}-\iota\left(\boldsymbol{x}_{i}\right)\right|}
$$

is well defined. Put $\psi:=\psi_{1} \times \psi_{2} \times \psi_{3}: B \rightarrow\left(S^{6 k-1}\right)^{\times 3}$ and consider

$$
\omega:=\psi^{*} \operatorname{vol}_{S^{6 k-1}}^{\times 3} \in \Omega_{D R}^{18 k-3}(B) .
$$

We can see that $\rho_{*} \omega \in \Omega_{D R}^{4 k}\left(\operatorname{Inj}_{6 k, 4 k-1}\right)$ is closed [25, Lemma 5.22]. $\operatorname{Inj}_{6 k, 4 k-1}$ is homeomorphic to the Stiefel manifold $V_{6 k, 4 k-1}$ of $(4 k-1)$-frames in $\mathbb{R}^{6 k}$, and hence 
$H_{D R}^{4 k}\left(\operatorname{Inj}_{6 k, 4 k-1}\right)=0$. Therefore, we can find $\mu \in \Omega_{D R}^{4 k-1}\left(\operatorname{Inj}_{6 k, 4 k-1}\right)$ such that

$$
\rho_{*} \omega=d \mu .
$$

The correction term $c: \mathcal{K}_{6 k, 4 k-1} \rightarrow \mathbb{R}$ is defined by

$$
c(f):=\frac{1}{6} \int_{\mathbb{R}^{4 k-1}}(d f)^{*} \mu \in \mathbb{R},
$$

where $d f: \mathbb{R}^{4 k-1} \rightarrow \operatorname{Inj}_{6 k, 4 k-1}$ is defined by the differential $x \mapsto d f_{x}$.

Let $C_{4,0}(f)$ and $C_{3,1}(f)$ be the fibers of $\pi_{X}: C_{4,0} \rightarrow \mathcal{K}_{6 k, 4 k-1}$ and $\pi_{Y}: C_{3,1} \rightarrow$ $\mathcal{K}_{6 k, 4 k-1}$ over $f$. These fibers are finite-dimensional configuration spaces with $\operatorname{dim} C_{4,0}(f)=\operatorname{deg} \omega_{X}$ and $\operatorname{dim} C_{3,1}(f)=\operatorname{deg} \omega_{Y} \cdot \mathcal{H}(f)$ is calculated as

$$
\mathcal{H}(f)=\frac{1}{2} \int_{C_{4,0}(f)} \omega_{X}+\frac{1}{6} \int_{C_{3,1}(f)} \omega_{Y}+c(f) .
$$

Theorem $4.3([\mathbf{2 5}])$. If $\operatorname{vol}_{S^{N-1}}(N=6 k, 4 k-1)$ are (anti-)invariant, then the 0 form $\mathcal{H}:=I(H)+c: \mathcal{K}_{6 k, 4 k-1} \rightarrow \mathbb{R}$ is closed and induces a group isomorphism $\pi_{0}\left(\mathcal{K}_{6 k, 4 k-1}\right) \cong \mathbb{Z}$. In particular, $\mathcal{H}$ is a $\mathbb{Z}$-valued invariant.

In [25] Theorem 4.3 is proved by evaluating $\mathcal{H}$ over a generator of $\pi_{0}\left(\mathcal{K}_{6 k, 4 k-1}\right)$ given by the spinning construction $[\mathbf{5}, \mathbf{2 4}]$. The computation in $\S 3$ gives an alternative proof.

To simplify the computations below, we will take the (anti-)invariant volume forms $\operatorname{vol}_{S^{N-1}}(N=6 k, 4 k-1)$ so that their supports are contained in small neighborhoods of the poles $\pm e_{N}:=(0, \ldots, 0, \pm 1) \in \mathbb{R}^{N}$. We call such a volume form a Dirac-type volume form. The following allows us to use such volume forms.

Proposition 4.4 ([25, Propositions 3.5, 3.6]). The invariant $\mathcal{H}$ is independent of the choice of the (anti-)invariant volume forms $\operatorname{vol}_{S^{N-1}}, N=6 k, 4 k-1$.

\section{Proofs of Theorems 2.5 and 2.9}

It is well known that the linking number of closed oriented submanifolds $M^{2 k-1} \sqcup$ $N^{2 k-1} \subset \mathbb{R}^{4 k-1}$ is an isotopy invariant and can be defined as

$$
l k(M, N):=\int_{M \times N} \varphi^{*} \operatorname{vol}_{S^{4 k-2}},
$$

where $\varphi: M \times N \rightarrow S^{4 k-2}$ is the generalized Gauss map given by

$$
\varphi(x, y):=\frac{y-x}{|y-x|} .
$$

If $M, N$ are in generic positions, then $p(M \sqcup N)$ is generically immersed in $\mathbb{R}^{4 k-2}$ and the pairs $(x, y) \in M \times N$ such that $p(\varphi(x, y))=\mathbf{0} \in \mathbb{R}^{4 k-2}$ form a 0-dimensional submanifold of $M \times N$. If, moreover, $\operatorname{vol}_{S^{4 k-2}}$ is Dirac-type (see the paragraph before 
Proposition 4.4), then

$$
\begin{aligned}
l k(M, N) & =\sum_{(x, y) \in(p \circ \varphi)^{-1}(\mathbf{0})} \int_{\begin{array}{c}
\text { a neighborhood } \\
\text { of }(x, y)
\end{array}} \varphi^{*} \operatorname{vol}_{S^{4 k-2}} \\
& =\left.\frac{1}{2} \sum_{(x, y) \in(p \circ \varphi)^{-1}(\mathbf{0})} \operatorname{deg} \varphi\right|_{\text {a neighborhood }} \text { of }(x, y)
\end{aligned}
$$

and each $\operatorname{deg} \varphi= \pm 1$. This is because the integrand $\varphi^{*} v l_{S^{4 k-2}}$ is zero outside neighborhoods of such pairs, and the integral of $\operatorname{vol}_{S^{4 k-2}}$ over one component of $\operatorname{supp}\left(\operatorname{vol}_{S^{4 k-2}}\right)$ is $1 / 2$. This interpretation gives us the following, which we use in $\S 6$.

Lemma 5.1. Let $M_{1}, M_{2}$ and $N_{1}, N_{2}$ be $(2 k-1)$-dimensional disjoint oriented submanifolds of $\mathbb{R}^{4 k-1}$. If the connected-sums $M_{1} \sharp M_{2}$ and $N_{1} \sharp N_{2}$ are taken in $\mathbb{R}^{4 k-1}$ so that $\left|p\left(M_{i}\right) \cap p\left(N_{j}\right)\right|(i, j=1,2)$ do not increase, then

$$
\sum_{i, j=1,2} l k\left(M_{i}, N_{j}\right)=l k\left(M_{1} \sharp M_{2}, N_{1} \sharp N_{2}\right) .
$$

For a single oriented submanifold $L^{2 k-1} \subset \mathbb{R}^{4 k-1}$, a similar formula to (5.1) does not give rise to an isotopy invariant, but if $L$ is almost planar, then such a function can be computed by counting $(x, y) \in \operatorname{Conf}_{2}^{\circ}(L)$ with $p(\varphi(x, y))=\mathbf{0}$.

Definition 5.2. Let $L^{2 k-1} \subset \mathbb{R}^{4 k-1}$ be a generic closed oriented submanifold such that $p(L) \subset \mathbb{R}^{4 k-2}$ is a generically immersed manifold. Define the writhe $w(L)$ of $L$ by

$$
\begin{aligned}
w(L) & =\sum_{(x, y) \in(p \circ \varphi)^{-1}(\mathbf{0})} \int_{\begin{array}{c}
\text { a neighborhood } \\
\text { of }(x, y)
\end{array}} \varphi^{*} \operatorname{vol}_{S^{4 k-2}} \\
& =\left.\frac{1}{2} \sum_{(x, y) \in(p \circ \varphi)^{-1}(\mathbf{0})} \operatorname{deg} \varphi\right|_{\text {a neighborhood. }} .
\end{aligned}
$$

For an almost planar $f \in \mathcal{K}_{6 k, 4 k-1}$, denote by $f^{\delta}$ the embedding obtained by a scaling in the $x_{6 k}$-direction so that $f^{\delta}\left(\mathbb{R}^{4 k-1}\right) \subset \mathbb{R}^{6 k-1} \times[0, \delta]$ (we often abbreviate $f^{\delta}$ as $\left.f\right)$. $L_{i}^{\epsilon}$ 's remain unchanged for any $\delta$. We compute $I(X)\left(f^{\delta}\right), I(Y)\left(f^{\delta}\right)$, and $c\left(f^{\delta}\right)$ in the limit $\delta \rightarrow 0$.

Proposition 5.3 (cf. [16, Proposition 4.3]). Suppose $\operatorname{vol}_{S^{N-1}}(N=6 k, 4 k-1)$ are Dirac-type. If $f \in \mathcal{K}_{6 k, 4 k-1}$ is almost planar and generic so that $l k\left(L_{i}^{\epsilon}, L_{j}^{\epsilon^{\prime}}\right)$ and $w\left(L_{i}^{\epsilon}\right)$ can be calculated by (5.1) and (5.2), then

$$
\lim _{\delta \rightarrow 0} I(X)\left(f^{\delta}\right)=\frac{1}{2} \sum_{(i, \epsilon)<\left(j, \epsilon^{\prime}\right)}(-1)^{\epsilon+\epsilon^{\prime}} l k\left(L_{i}^{\epsilon}, L_{j}^{\epsilon^{\prime}}\right)+\frac{1}{4} \sum_{i, \epsilon} w\left(L_{i}^{\epsilon}\right) .
$$

Proof. A configuration $\vec{\xi}=\left(\boldsymbol{\xi}_{1}, \ldots, \boldsymbol{\xi}_{4}\right) \in C_{4,0}(f)$ can non-trivially contribute to $I(X)$ only if $\vec{\xi} \in \varphi_{X}^{-1}\left(\operatorname{supp}\left(\operatorname{vol}_{S^{6 k-1}}^{\times 2} \times \operatorname{vol}_{S^{4 k-2}}\right)\right)$. Since $\operatorname{vol}_{S^{N-1}}$ are Dirac-type, such a $\vec{\xi}$ must be in a neighborhood of $\varphi_{X}^{-1}\left( \pm e_{6 k}, \pm e_{6 k}, \pm e_{4 k-1}\right)$, where $e_{N}:=(0, \ldots, 0,1) \in$ $S^{N-1}$.

If $\delta$ is sufficiently close to 0 , then no vectors tangent to $f\left(\mathbb{R}^{4 k-1}\right)$ point $\operatorname{supp}\left(\operatorname{vol}_{S^{6 k-1}}\right)$. Thus $\vec{\xi}$ can be in $\varphi_{X}^{-1}\left(\operatorname{supp}\left(\operatorname{vol}_{S^{6 k-1}}^{\times 2} \times \operatorname{vol}_{S^{4 k-2}}\right)\right)$ in the limit $\delta \rightarrow 0$ 
only if $\left(\boldsymbol{\xi}_{1}, \boldsymbol{\xi}_{2}\right) \in N\left(L_{i}^{\epsilon}\right) \times N\left(L_{i}^{\epsilon+1}\right)$ and $\left(\boldsymbol{\xi}_{3}, \boldsymbol{\xi}_{4}\right) \in N\left(L_{j}^{\epsilon^{\prime}}\right) \times N\left(L_{j}^{\epsilon^{\prime}+1}\right)$ for some $i, j$, possibly $i=j$ (recall that $N\left(L_{i}^{\epsilon}\right) \subset \mathbb{R}^{4 k-1}$ are closed disjoint tubular neighborhoods of $\left.L_{i}^{\epsilon}\right)$. For $(s, t)=(1,2),(4,3)$, and any $\boldsymbol{\xi}_{t} \in N\left(L_{i}^{\epsilon}\right)$, we always find $\boldsymbol{\xi}_{s} \in N\left(L_{i}^{\epsilon+1}\right)$ such that $p\left(f\left(\boldsymbol{\xi}_{s}\right)-f\left(\boldsymbol{\xi}_{t}\right)\right)$ is close to $\mathbf{0}$. Therefore, finding such a $\vec{\xi}=\left(\boldsymbol{\xi}_{1}, \ldots, \boldsymbol{\xi}_{4}\right) \in$ $\varphi_{X}^{-1}\left( \pm e_{6 k}, \pm e_{6 k}, \pm e_{4 k-1}\right)$ is equivalent to finding $\left(\boldsymbol{\xi}_{2}, \boldsymbol{\xi}_{3}\right)$ satisfying $p\left(\varphi_{23}^{\eta}\left(\boldsymbol{\xi}_{2}, \boldsymbol{\xi}_{3}\right)\right)=\mathbf{0}$. By our assumption on $f$, the set of such $\vec{\xi}$ 's is a 0-dimensional submanifold of $C_{4,0}(f)$, each component of whose neighborhood is mapped homeomorphically into a component of $\operatorname{supp}\left(\operatorname{vol}_{S^{6 k-1}}^{\times 2} \times \operatorname{vol}_{S^{4 k-2}}\right)$ via $\varphi_{X}$. The integral of $\omega_{X}$ over such a component is $\pm(1 / 2)^{3}$, where the sign is the local degree of $\varphi_{X}$ at $\vec{\xi}$ - that is, the determinant of the Jacobian $J\left(\varphi_{X}\right)_{\vec{\xi}}$. The sum of these degrees would amount to linking numbers by $(5.1)$.

To compute $\operatorname{deg} \varphi_{X}$ at each $\vec{\xi} \in \varphi_{X}^{-1}\left( \pm e_{6 k}, \pm e_{6 k}, \pm e_{4 k-1}\right)$, we recall from $[\mathbf{1 0}, \mathbf{1 1}]$ the local model for two-fold self-intersection. Let $g \in \mathcal{I}_{6 k-1,4 k-1}$ be a generic immersion, and let $q=g\left(p_{1}\right)=g\left(p_{2}\right)$ be a transverse two-fold self-intersection point. In some local coordinates centered at $p_{1}, p_{2}$, and $q, g$ is given by

$$
\begin{aligned}
g\left(x_{1}, \ldots, x_{4 k-1}\right) & =\left(x_{1}, \ldots, x_{2 k-1}, x_{2 k}, \ldots, x_{4 k-1}, 0, \ldots, 0\right) & & \text { near } p_{1}, \\
g\left(y_{1}, \ldots, y_{4 k-1}\right) & =\left(y_{1}, \ldots, y_{2 k-1}, 0, \ldots, 0, \quad y_{2 k}, \ldots, y_{4 k-1}\right) & & \text { near } p_{2} .
\end{aligned}
$$

Now consider a configuration $\vec{\xi} \in C_{4,0}(f)$ such that

$$
\left\{\begin{array}{l}
\boldsymbol{\xi}_{1} \in L_{i}^{1}, \boldsymbol{\xi}_{2} \in L_{i}^{0}, \boldsymbol{\xi}_{3} \in L_{j}^{0} \text { and } \boldsymbol{\xi}_{4} \in L_{j}^{1}, \\
\varphi_{X}(\vec{\xi})=\left(-e_{6 k},-e_{6 k}, e_{4 k-1}\right)
\end{array}\right.
$$

(Figure 5.1, left). Suppose that $\left(\boldsymbol{\xi}_{2}, \boldsymbol{\xi}_{3}\right)$ is a positive crossing - that is, $\left.\operatorname{deg} \varphi_{23}^{\eta}\right|_{\left(\boldsymbol{\xi}_{2}, \boldsymbol{\xi}_{3}\right)}=$ +1 . Then we can choose some local coordinates $\boldsymbol{x}, \boldsymbol{y}$, and $\boldsymbol{z}$ centered at $\boldsymbol{\xi}_{1}, \boldsymbol{\xi}_{2}$, and $\boldsymbol{\xi}_{4}$ such that $\boldsymbol{\xi}_{3}=(0, \ldots, 0,1)$ in the $\boldsymbol{y}$-coordinate (the same coordinate as for $\left.\boldsymbol{\xi}_{2}\right)$, and we can also choose local coordinates in $\mathbb{R}^{6 k}$ in which $f$ is given by

$$
\begin{aligned}
& f(\boldsymbol{x})=\left(x_{1}, \ldots, x_{2 k-1}, x_{2 k}, \ldots, x_{4 k-1}, 0, \ldots, 0, \quad 1\right) \\
& f(\boldsymbol{y})=\left(y_{1}, \ldots, y_{2 k-1}, 0, \ldots, 0, \quad y_{2 k}, \ldots, y_{4 k-1}, 0\right) \\
& \text { near } \boldsymbol{\xi}_{1},
\end{aligned}
$$

and

$$
\begin{array}{llll}
f(\boldsymbol{y}) & =\left(0, \ldots, 0, \quad y_{1}, \ldots, y_{2 k-1}, y_{2 k}, \ldots, y_{4 k-2}, y_{4 k-1}, 0,\right. & 1 & \text { near } \boldsymbol{\xi}_{3}, \\
f(\boldsymbol{z}) & =\left(z_{1}, \ldots, z_{2 k-1}, 0, \ldots, 0, \quad z_{2 k}, \ldots, z_{4 k-2}, 1, \quad z_{4 k-1}, 0\right) & \text { near } \boldsymbol{\xi}_{4} .
\end{array}
$$

Then by Lemma 2.3, as oriented manifolds, $L_{*}^{\epsilon}$ 's are given by

- $L_{i}^{1} \cap(\boldsymbol{x}$-coordinate $)=+\mathbb{R}^{2 k-1} \times\{\mathbf{0}\}^{2 k}$,

- $L_{i}^{0} \cap(\boldsymbol{y}$-coordinate $)=+\mathbb{R}^{2 k-1} \times\{\mathbf{0}\}^{2 k}$,

- $L_{j}^{1} \cap(\boldsymbol{y}$-coordinate $)=\{\mathbf{0}\}^{2 k-1} \times\left(-\mathbb{R}^{2 k-1}\right) \times\{1\}$, and

- $L_{j}^{0} \cap(\boldsymbol{z}$-coordinate $)=\{\mathbf{0}\}^{2 k-1} \times\left(-\mathbb{R}^{2 k-1}\right) \times\{0\}$

(see Figure 5.2). Using this local model, we can compute the Jacobian $J\left(\varphi_{X}\right)_{\vec{\xi}}$ of

$$
\varphi_{X}: N\left(L_{i}^{1}\right) \times N\left(L_{i}^{0}\right) \times N\left(L_{j}^{1}\right) \times N\left(L_{j}^{0}\right) \rightarrow S^{6 k-1} \times S^{6 k-1} \times S^{4 k-2}
$$

at $\vec{\xi}$ explicitly. Let $e_{i}:=(0, \ldots, 0,1,0, \ldots, 0)$ be the $i$ th unit vector. With respect to the natural positive basis $e_{1}, \ldots, e_{16 k-4}$ of $T_{\vec{\xi}} \operatorname{Conf}_{4}\left(\mathbb{R}^{4 k-1}\right) \cong T_{\vec{\xi}} \mathbb{R}^{16 k-4}$ and the 


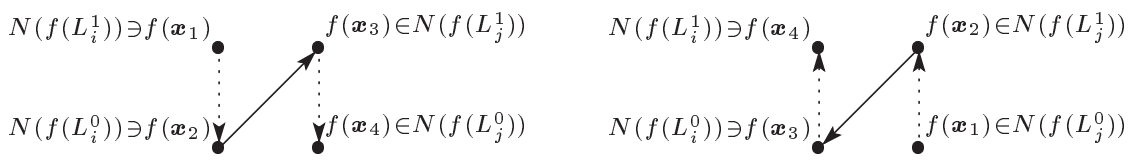

Figure 5.1: Two configurations with the same contributions to $l k\left(L_{i}^{0}, L_{j}^{1}\right)$; the left shows a neighborhood of $\vec{\xi}$ satisfying (5.3), and the right shows a neighborhood of $\overrightarrow{\xi^{\prime}}$ satisfying (5.4).

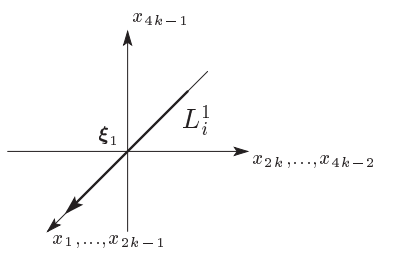

the $\boldsymbol{x}$-sheet

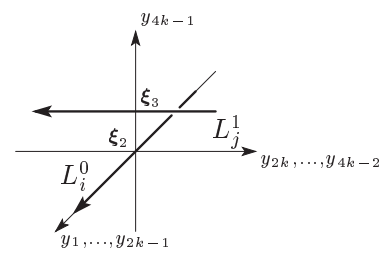

the $\boldsymbol{y}$-sheet

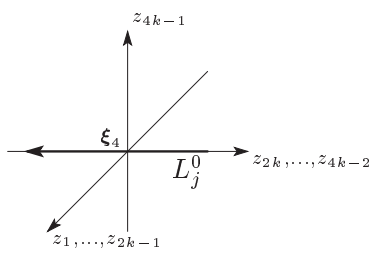

the $\boldsymbol{z}$-sheet

Figure 5.2: Local picture of $L_{i}^{0} \cup L_{i}^{1} \cup L_{j}^{0} \cup L_{j}^{1}$

natural positive bases of the tangent spaces of spheres

$$
e_{1}, \ldots, e_{6 k-1} \in T_{-e_{6 k}} S^{6 k-1} \text { and } e_{1}, \ldots, e_{4 k-2} \in T_{e_{4 k-1}} S^{4 k-2},
$$

following the "outward normal first" convention, $J\left(\varphi_{X}\right)_{\vec{\xi}}$ is given as in Appendix A and its determinant is -1 .

A local model for a negative crossing $\left(\boldsymbol{\xi}_{2}, \boldsymbol{\xi}_{3}\right)$ (namely, $\left.\operatorname{deg} \varphi_{23}^{\eta}\right|_{\left(\boldsymbol{\xi}_{2}, \boldsymbol{\xi}_{3}\right)}=-1$ ) is obtained from the above model by reversing the orientation of the $\boldsymbol{z}$-sheet, and, in this case, $J\left(\varphi_{X}\right)_{\vec{\xi}}=+1$. Thus the integral of $\omega_{X}$ over a neighborhood of $\vec{\xi}$ satisfying (5.3) with $\left.\operatorname{deg} \varphi_{23}^{\eta}\right|_{\left(\boldsymbol{\xi}_{2}, \boldsymbol{\xi}_{3}\right)}= \pm 1$ is $\mp(1 / 2)^{3}$, and by $(5.1)$ their sum is equal to $-l k\left(L_{i}^{0}, L_{j}^{1}\right) / 4$. By symmetry of $X$, the configurations near $\vec{\xi}^{\prime}$ satisfying

$$
\left\{\begin{array}{l}
\boldsymbol{\xi}_{1}^{\prime} \in L_{j}^{0}, \boldsymbol{\xi}_{2}^{\prime} \in L_{j}^{1}, \boldsymbol{\xi}_{3}^{\prime} \in L_{i}^{1} \text { and } \boldsymbol{\xi}_{4}^{\prime} \in L_{i}^{0}, \\
\varphi_{X}(\vec{\xi})=\left(e_{6 k}, e_{6 k},-e_{4 k-1}\right)
\end{array}\right.
$$

(see Figure 5.1, right) also contribute to $I(X)(f)$ by $-l k\left(L_{i}^{0}, L_{j}^{1}\right) / 4$. Thus a link $L_{i}^{0} \sqcup L_{j}^{1}$ contributes to $I(X)(f)$ by $-l k\left(L_{i}^{0}, L_{j}^{1}\right) / 2$.

A similar computation shows that $L_{i}^{0}$ and $L_{j}^{0}$ contribute to $I(X)(f)$ by $+l k\left(L_{i}^{0}, L_{j}^{0}\right) / 2$; we have the same Jacobian matrix as above, but in this case $\varphi_{34}^{\theta}(\vec{\xi})=$ $e_{6 k}$ and $\left(e_{1}, \ldots, e_{6 k-1}\right)$ represent the negative orientation of $T_{e_{6 k}} S^{6 k-1}$ and the sign of the degree changes. This observation shows that, in general, the link $L_{i}^{\epsilon} \sqcup L_{j}^{\epsilon^{\prime}}$, $(i, \epsilon) \neq\left(j, \epsilon^{\prime}\right)$, contributes to $I(X)(f)$ by $(-1)^{\epsilon+\epsilon^{\prime}} l k\left(L_{i}^{\epsilon}, L_{j}^{\epsilon^{\prime}}\right) / 2$.

In the case $(i, \epsilon)=\left(j, \epsilon^{\prime}\right)$, if $f$ is generic so that $w\left(L_{i}^{\epsilon}\right)$ can be calculated by (5.2), then the same computation as above shows that the configurations in $N\left(L_{i}^{\epsilon+1}\right) \times$ $N\left(L_{i}^{\epsilon}\right) \times N\left(L_{i}^{\epsilon}\right) \times N\left(L_{i}^{\epsilon+1}\right)$ contributes to $I(X)(f)$ by $+w\left(L_{i}^{\epsilon}\right) / 4$ (no sign appears in this case since $\left.\epsilon=\epsilon^{\prime}\right)$. 
Lemma 5.4. Let $f \in \mathcal{K}_{6 k, 4 k-1}$ be almost planar, and suppose that $\operatorname{vol}_{S^{6 k-1}}$ is Diractype. Then

$$
\lim _{\delta \rightarrow 0} I(Y)\left(f^{\delta}\right)=\lim _{\delta \rightarrow 0} I(Y)\left(f_{S}^{\delta}\right) \quad \text { and } \quad \lim _{\delta \rightarrow 0} c\left(f^{\delta}\right)=\lim _{\delta \rightarrow 0} c\left(f_{S}^{\delta}\right) .
$$

Proof. The function $c: \mathcal{K}_{6 k, 4 k-1} \rightarrow \mathbb{R}$ is defined and continuous on the space of immersions, because $c(f)$ is determined by the differential of $f$ (see $\S 4.2$ ). Thus $c\left(f^{\delta}\right)$ continuously depends on $\delta$ including $\delta=0$ (then $f$ and $f_{S}$ collapse down to an immersion $\left.f^{0}=f_{S}^{0}=p \circ f\right)$, and hence $\lim _{\delta \rightarrow 0} c(f)=c(p \circ f)=\lim _{\delta \rightarrow 0} c\left(f_{S}\right)$.

The above observation also implies that $\lim _{\delta \rightarrow 0} I(Y)\left(f^{\delta}\right)$ exists, because $I(Y)\left(f^{\delta}\right)=6 \mathcal{H}\left(f^{\delta}\right)-3 I(X)\left(f^{\delta}\right)-6 c\left(f^{\delta}\right)$ and the limit of the right-hand side exists by Proposition 5.3, the existence of $\lim c\left(f^{\delta}\right)$, and the fact that $\mathcal{H}$ is an isotopy invariant. To compute $\lim _{\delta \rightarrow 0} I(Y)\left(f^{\delta}\right)$, we may assume that $\delta<1$. Choose open neighborhoods $f\left(N\left(L_{i}^{0}\right)\right) \subset U_{i}^{\prime} \subset V_{i}^{\prime}$ in $\mathbb{R}^{6 k-1}$ (we are assuming $f$ is almost planar) so that $\overline{U_{i}^{\prime}} \subset V_{i}^{\prime}, V_{i}^{\prime} \cap V_{j}^{\prime}=\emptyset(i \neq j)$, and $f\left(N\left(L_{i}^{1}\right)\right) \subset U_{i}:=U_{i}^{\prime} \times[-1,1]$ (Figure 5.3). Let $C_{3,1}^{(1)}(f)$ be the subspace of $C_{3,1}(f)$ consisting of $\vec{x} \in C_{3,1}(f)$ with $\boldsymbol{x}_{4} \in V_{i}:=$ $V_{i}^{\prime} \times[-1,1]$ for some $i \in S$. Now we compare the integrals of $\omega_{Y}$ over $C_{3,1}^{(1)}(f)$ and

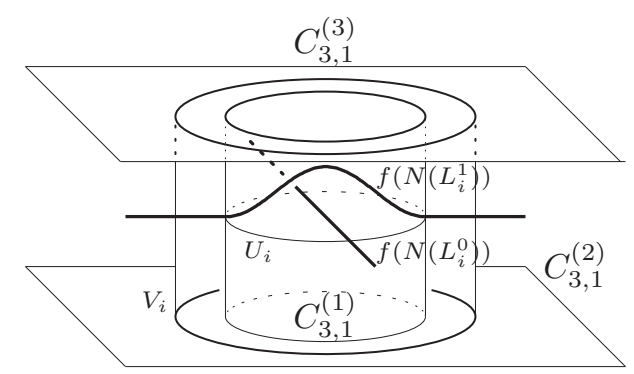

Figure 5.3: The places in which $\boldsymbol{x}_{4}$ is $\left(\vec{x} \in C_{3,1}^{(l)}, l=1,2,3\right)$

$C_{3,1}^{(1)}\left(f_{S}\right)$. Since $\operatorname{vol}_{S^{6 k-1}}$ is Dirac-type, we only need to consider the set of $\vec{x}$ 's with $\boldsymbol{x}_{1}, \boldsymbol{x}_{2}, \boldsymbol{x}_{3} \in f^{-1}\left(V_{i}\right)$ for the same $i$ as for $\boldsymbol{x}_{4}$, since $\omega_{Y}$ vanishes on other $\vec{x}$ 's. The local diffeomorphism $\Phi: C_{3,1}^{(1)}(f) \rightarrow C_{3,1}^{(1)}\left(f_{S}\right)$,

$$
\Phi\left(\left(\boldsymbol{x}_{1}, \boldsymbol{x}_{2}, \boldsymbol{x}_{3}\right) ; \boldsymbol{x}_{4}\right):=\left(\left(\boldsymbol{x}_{1}, \boldsymbol{x}_{2}, \boldsymbol{x}_{3}\right) ; \iota\left(\boldsymbol{x}_{4}\right)\right),
$$

reverses the orientation, while $\Phi^{*} \omega_{Y}=-\omega_{Y}$ since $\psi_{j}^{\theta} \circ \Phi=\iota \circ \psi_{j}^{\theta}$ and $f_{S}=\iota \circ f$ on $f^{-1}\left(V_{i}\right)(i \in S)$ and $\iota^{*} \operatorname{vol}_{S^{6 k-1}}=-\operatorname{vol}_{S^{6 k-1}}$ (because $\operatorname{vol}_{S^{6 k-1}}$ is anti-invariant). Thus the integrations of $\omega_{Y}$ over $C_{3,1}^{(1)}(f)$ and $C_{3,1}^{(1)}\left(f_{S}\right)$ are equal to each other.

Next, consider the subspace $C_{3,1}^{(2)}(f)$ of $C_{3,1}(f)$ consisting of $\vec{x}$ with $\boldsymbol{x}_{4} \in \mathbb{R}^{6 k-1} \times$ $[-1,1]$ but $\boldsymbol{x}_{4} \notin V_{i}$ for any $i \in S$. Since $\operatorname{vol}_{S^{6 k-1}}$ is Dirac-type and since $\boldsymbol{x}_{4} \notin V_{i}$ for any $i \in S, \vec{x} \in C_{3,1}^{(2)}(f)$ can non-trivially contribute to $I(Y)$ only if $\boldsymbol{x}_{j}(j=1,2,3)$ is not contained in any $L_{i}^{1}(i \in S)$. Since $f=f_{S}$ outside $L_{i}^{1}(i \in S)$, there is no difference between $\omega_{Y}$ 's on $C_{3,1}^{(2)}(f)$ and $C_{3,1}^{(2)}\left(f_{S}\right)$, and the integrals of $\omega_{Y}$ over $C_{3,1}^{(2)}(f)$ and $C_{3,1}^{(2)}\left(f_{S}\right)$ are also the same.

Finally consider the subspace $C_{3,1}^{(3)}(f)$ of $C_{3,1}(f)$ consisting of $\vec{x}$ with $\boldsymbol{x}_{4} \notin \mathbb{R}^{6 k-1} \times$ $[-1,1]$. If $\delta>0$ is small enough, then under the diffeomorphism $C_{3,1}^{(3)}(f) \rightarrow C_{3,1}^{(3)}\left(f_{S}\right)$ 
given by $\vec{x} \mapsto \vec{x}$, the differences between the vectors $\psi_{i}^{\theta}(\vec{x})(i=1,2,3)$ are small. This is because $f$ differs from $f_{S}$ only near $N\left(L_{i}^{1}\right)$ and the difference is small relative to $\left|\boldsymbol{x}_{4}\right|$. Thus the difference between the integrals of $\omega_{Y}$ over $C_{3,1}^{(3)}(f)$ and $C_{3,1}^{(3)}\left(f_{S}\right)$ converges to 0 in the limit $\delta \rightarrow 0$.

Proof of Theorem 2.5. Any $f$ with $p \circ f$ generic can be transformed by an ambient isotopy of $\mathbb{R}^{4 k-1}$ so that $f$ satisfies the condition in Proposition 5.3 without changing the isotopy class of $\bigcup L_{i}^{\epsilon}$. Thus we may assume that $f$ satisfies the condition in Proposition 5.3. Notice

$$
\mathcal{H}(f)-\mathcal{H}\left(f_{S}\right)=\frac{I(X)(f)-I(X)\left(f_{S}\right)}{2}+\frac{I(Y)(f)-I(Y)\left(f_{S}\right)}{6}+\left(c(f)-c\left(f_{S}\right)\right) .
$$

The left-hand side does not depend on $\delta$. In the limit $\delta \rightarrow 0$ ( $p \circ f$ remains unchanged), the first term of the right-hand side is computed in Proposition 5.3 and gives the righthand side of (2.1). The second and the third terms converge to zero by Lemma 5.4.

Proof of Theorem 2.9. Choose three components $A_{1}, A_{2}, A_{3}$ out of $A=A_{1} \sqcup \cdots \sqcup$ $A_{m}, m \geqslant 3$. Let $W_{T}(\mathcal{H})(f):=\mathcal{H}\left(f_{T}\right)-\mathcal{H}\left(f_{T \cup\{1\}}\right)$ for any $T \subset\{2,3\}$. Then by $(2.2)$,

$$
2 W_{T}(\mathcal{H})(f)=\sum_{j \neq 1 ; \epsilon, \epsilon^{\prime}=0,1}(-1)^{\epsilon+\epsilon^{\prime}} l k\left(L_{1}^{\epsilon}\left(f_{T}\right), L_{j}^{\epsilon^{\prime}}\left(f_{T}\right)\right) .
$$

Because $L_{j}^{\epsilon}\left(f_{T}\right)=L_{i}^{\epsilon+1}(f)$ if $j \in T$ and $L_{j}^{\epsilon}\left(f_{T}\right)=L_{j}^{\epsilon}(f)$ otherwise,

$$
\begin{aligned}
2 W_{\emptyset}(\mathcal{H})(f) & =\sum_{\epsilon, \epsilon^{\prime}}(-1)^{\epsilon+\epsilon^{\prime}}\left(l k\left(L_{1}^{\epsilon}, L_{2}^{\epsilon^{\prime}}\right)+l k\left(L_{1}^{\epsilon}, L_{3}^{\epsilon^{\prime}}\right)\right)+\sum_{j \geqslant 4 ; \epsilon, \epsilon^{\prime}}(-1)^{\epsilon+\epsilon^{\prime}} l k\left(L_{1}^{\epsilon}, L_{j}^{\epsilon^{\prime}}\right), \\
2 W_{\{2\}}(\mathcal{H})(f) & =\sum_{\epsilon, \epsilon^{\prime}}(-1)^{\epsilon+\epsilon^{\prime}}\left(-l k\left(L_{1}^{\epsilon}, L_{2}^{\epsilon^{\prime}}\right)+l k\left(L_{1}^{\epsilon}, L_{3}^{\epsilon^{\prime}}\right)\right)+\sum_{j \geqslant 4 ; \epsilon, \epsilon^{\prime}}(-1)^{\epsilon+\epsilon^{\prime}} l k\left(L_{1}^{\epsilon}, L_{j}^{\epsilon^{\prime}}\right), \\
2 W_{\{3\}}(\mathcal{H})(f) & =\sum_{\epsilon, \epsilon^{\prime}}(-1)^{\epsilon+\epsilon^{\prime}}\left(l k\left(L_{1}^{\epsilon}, L_{2}^{\epsilon^{\prime}}\right)-l k\left(L_{1}^{\epsilon}, L_{3}^{\epsilon^{\prime}}\right)\right)+\sum_{j \geqslant 4 ; \epsilon, \epsilon^{\prime}}(-1)^{\epsilon+\epsilon^{\prime}} l k\left(L_{1}^{\epsilon}, L_{j}^{\epsilon^{\prime}}\right), \\
2 W_{\{2,3\}}(\mathcal{H})(f) & =\sum_{\epsilon, \epsilon^{\prime}}(-1)^{\epsilon+\epsilon^{\prime}}\left(-l k\left(L_{1}^{\epsilon}, L_{2}^{\epsilon^{\prime}}\right)-l k\left(L_{1}^{\epsilon}, L_{3}^{\epsilon^{\prime}}\right)\right)+\sum_{j \geqslant 4 ; \epsilon, \epsilon^{\prime}}(-1)^{\epsilon+\epsilon^{\prime}} l k\left(L_{1}^{\epsilon}, L_{j}^{\epsilon^{\prime}}\right) .
\end{aligned}
$$

Substituting into $V_{3}(\mathcal{H})(f)=\sum_{T \subset\{2,3\}}(-1)^{|T|} W_{T}(\mathcal{H})(f)$, we obtain $V_{3}(\mathcal{H})(f)=0$.

\section{Proof of Theorem 2.11}

\subsection{Well-definedness and invariance of $E$}

Suppose $g \in \mathcal{I}_{6 k-1,4 k-1}$ is generic and liftable, and let $f, f^{\prime} \in \mathcal{K}_{6 k, 4 k-1}$ be lifts of $g$. We can transform $f^{\prime}$ by an isotopy in the $x_{6 k}$-direction (without changing $p \circ f^{\prime}$ ) so that $f^{\prime}=f_{S}$ for some index set $S$ of the self-intersection of $g$. Then (2.1) implies that $E(g)$ does not depend on the choice of $f$.

Let $g_{t} \in \mathcal{I}_{6 k-1,4 k-1}(t \in[0,1])$ be a generic regular homotopy with each $g_{t}$ liftable. We show that, for any $t_{0} \in[0,1], g_{t}$ can be lifted to an isotopy $f_{t} \in \mathcal{K}_{6 k, 4 k-1}$-namely, $g_{t}=p \circ f_{t}$-in an open neighborhood of $t_{0}$. Let $f_{t_{0}}$ be a lift of $g_{t_{0}}$. Then $f_{t_{0}}$ can be 
written as $f_{t_{0}}=\left(g_{t_{0}}, h\right)$ by using some $h: \mathbb{R}^{4 k-1} \rightarrow \mathbb{R}$. Define $G_{t}: \operatorname{Conf}_{2}^{\circ}\left(\mathbb{R}^{4 k-1}\right) \rightarrow$ $\mathbb{R}^{6 k-1}$ by

$$
G_{t}(\boldsymbol{x}, \boldsymbol{y}):=g_{t}(\boldsymbol{x})-g_{t}(\boldsymbol{y}) .
$$

The first projection $\operatorname{Conf}_{2}^{\circ}\left(\mathbb{R}^{4 k-1}\right) \rightarrow \mathbb{R}^{4 k-1}$ restricts to a diffeomorphism $G_{t}^{-1}(\mathbf{0}) \cong$ $g_{t}^{-1}\left(A_{t}\right)$, where $A_{t}$ is the self-intersection of $g_{t}$. Since $g_{t}$ is a generic regular homotopy, $G_{t}^{-1}(\mathbf{0})$ gives an isotopy of a closed submanifold of Conf ${ }_{2}^{\circ}\left(\mathbb{R}^{4 k-1}\right)$. Because $f_{t_{0}} \in$ $\mathcal{K}_{6 k, 4 k-1}$, there exists an open neighborhood $W$ of $G_{t_{0}}^{-1}(\mathbf{0})$ such that $h(\boldsymbol{x}) \neq h(\boldsymbol{y})$ for any $(\boldsymbol{x}, \boldsymbol{y}) \in W$. The compactness of $G_{t}^{-1}(\mathbf{0})$ (for any $t$ ) implies that there exists $\epsilon>0$ such that $G_{t}^{-1}(\mathbf{0}) \subset W$ for $\left|t-t_{0}\right|<\epsilon$. Then $f_{t}:=\left(g_{t}, h\right): \mathbb{R}^{4 k-1} \rightarrow \mathbb{R}^{6 k}$ is in $\mathcal{K}_{6 k, 4 k-1}$ for $\left|t-t_{0}\right|<\epsilon$ and is a lift of $g_{t}$.

Because $\mathcal{H}\left(f_{t}\right)$ is constant and the linking part of (2.4) is invariant unless the double point set varies, $E\left(g_{t}\right)$ is also constant. Thus for any $t_{0}$ there exists $\epsilon>0$ such that $E\left(g_{t}\right)$ is constant on $\left(t_{0}-\epsilon, t_{0}+\epsilon\right)$, and hence $E\left(g_{t}\right)$ is constant on $[0,1]$.

Remark 6.1. By Proposition 5.3 and (2.4), for a generic liftable $g \in \mathcal{I}_{6 k-1,4 k-1}$,

$$
E(g)=\lim _{\delta \rightarrow 0}\left(\frac{1}{6} I(Y)\left(f^{\delta}\right)+c\left(f^{\delta}\right)+\frac{1}{8} \sum_{i, \epsilon} w\left(L_{i}^{\epsilon}\right)\right) .
$$

This gives a geometric interpretation of $I(Y)$ (added by $c$ and the writhes), and is a higher-dimensional analogue to [16, Definition 5.4].

\subsection{Local models of non-generic self-intersections}

As explained in $[\mathbf{1 0}, \mathbf{1 1}]$, the set of generic immersions $g: \mathbb{R}^{4 k-1} \rightarrow \rightarrow \mathbb{R}^{6 k-1}$ is an open dense subspace of $\mathcal{I}_{6 k-1,4 k-1}$ and the complement is a stratified hypersurface. To characterize an invariant of generic immersions, we must study its jumps at nongeneric strata. The codimension 1 strata (in $\mathcal{I}_{6 k-1,4 k-1}$ ) consist of immersions with a single generic self-tangency point or a single generic triple point [11, Lemma 3.4]. The local picture of the versal deformation $[\mathbf{1 0}, \S 5.3],[\mathbf{1 1}, \S 3.2]$ of an immersion with a self-tangency or a triple point is given in $[\mathbf{1 0}, \mathbf{1 1}]$.

Proposition 6.2 ([11, Lemma 3.5]). Let $g_{0} \in \mathcal{I}_{6 k-1,4 k-1}$ be an immersion with a single generic self-tangency point. Then the versal deformation $g_{t}$ of $g_{0}$ is constant far from the self-tangency point, and in some local coordinates near the self-tangency point, $g_{t}$ is given by

$$
\begin{aligned}
& g_{t}(\boldsymbol{x})=\left(x_{1}, \ldots, x_{2 k}, x_{2 k+1}, \ldots, x_{4 k-1}, 0, \quad 0, \quad \ldots, 0\right), \\
& g_{t}(\boldsymbol{y})=\left(y_{1}, \ldots, y_{2 k}, 0, \quad \ldots, 0, \quad Q\left(y_{1}, \ldots, y_{2 k}\right)+t, y_{2 k+1}, \ldots, y_{4 k-1}\right),
\end{aligned}
$$

where $Q$ is a non-degenerate quadratic form on $2 k$ variables.

We say a self-tangency point definite (resp. indefinite) if the quadratic form $Q$ is definite (resp. indefinite).

Proposition 6.3 ([11, Lemma 3.6]). Let $g_{0} \in \mathcal{I}_{6 k-1,4 k-1}$ be an immersion with a single generic triple point. Then the versal deformation $g_{t}$ of $g_{0}$ is constant far from 
the triple point, and in some local coordinates near the triple point, $g_{t}$ is given by

$$
\begin{aligned}
g_{t}(\boldsymbol{x}) & =\left(x_{1}, \ldots, x_{2 k-1}, x_{2 k}, x_{2 k+1}, \ldots, x_{4 k-1}, 0, \quad 0, \quad \ldots, 0\right), \\
g_{t}(\boldsymbol{y}) & =\left(y_{1}, \ldots, y_{2 k-1}, 0, \quad 0, \quad \ldots, 0, \quad y_{2 k}, \quad y_{2 k+1}, \ldots, y_{4 k-1}\right), \\
g_{t}(\boldsymbol{z}) & =\left(\begin{array}{lll}
0, \ldots, 0, \quad z_{2 k}, z_{1}, \quad \ldots, z_{2 k-1}, z_{2 k}-t, z_{2 k+1}, \ldots, z_{4 k-1}
\end{array}\right) .
\end{aligned}
$$

\subsection{The jump of $E$ at a non-generic liftable immersion}

Suppose that $g_{0} \in \mathcal{I}_{6 k-1,4 k-1}$ is liftable and has a single generic self-tangency point or a single generic triple point, and let $g_{t}$ be its versal deformation.

We show that $g_{t}$ is liftable for $|t|$ small. Let $f_{0}=\left(g_{0}, h\right)$ be a lift of $g_{0}$ and $f_{t}:=$ $\left(g_{t}, h\right) \in \mathcal{I}_{6 k-1,4 k-1}$. Similarly to the argument in $\S 6.1$, choose an open neighborhood $W$ of $G_{0}^{-1}(\mathbf{0})$ such that $h(\boldsymbol{x}) \neq h(\boldsymbol{y})$ for any $(\boldsymbol{x}, \boldsymbol{y}) \in W$. Then there exists $\epsilon>0$ such that $G_{t}^{-1}(\mathbf{0}) \subset W$ for $|t|<\epsilon$; this follows from the explicit description of the change of the multiple point set of $g_{t}$ (see below). Thus $f_{t} \in \mathcal{K}_{6 k, 4 k-1}$ for $|t|<\epsilon$ and is a lift of $g_{t}$.

By the definition (2.4) of the invariant $E$, its jump $E\left(g_{t}\right)-E\left(g_{-t}\right)(t \neq 0)$ is described by the change of linking numbers of $L_{i}^{\epsilon}$ 's because $\mathcal{H}\left(f_{t}\right)$ remains unchanged.

\subsubsection{Definite self-tangencies}

First, we study the jump of $E$ at a positive definite self-tangency point (the argument needs no change for the negative definite case). It is clear from Proposition 6.2 that in some local coordinate near the tangency point, the double point set is given by

$$
\begin{array}{ll}
K^{0}=\left\{\left(x_{1}, \ldots, x_{2 k}, \mathbf{0}^{2 k-1}\right) \in \mathbb{R}^{4 k-1} \mid x_{1}^{2}+\cdots+x_{2 k}^{2}=-t\right\} & \text { in the } \boldsymbol{x} \text {-sheet, } \\
K^{1}=\left\{\left(y_{1}, \ldots, y_{2 k}, \mathbf{0}^{2 k-1}\right) \in \mathbb{R}^{4 k-1} \mid y_{1}^{2}+\cdots+y_{2 k}^{2}=-t\right\} & \text { in the } \boldsymbol{y} \text {-sheet, }
\end{array}
$$

which is empty when $t>0$ and the trivial link when $t<0$. This link $K^{0} \sqcup K^{1}$ is separated from the other links since each $K^{\epsilon}$ is contained in a small open set that intersects no other components of the double point set. Thus we have the following.

Lemma 6.4. If $g_{0}$ has a definite self-tangency point, then $E\left(g_{t}\right)=E\left(g_{-t}\right)$.

\subsubsection{Indefinite self-tangencies}

Next, suppose that $g_{0}$ is liftable and has an indefinite self-tangency point. Let $0<$ $\lambda<2 k$ be the index of $Q$. By Proposition 6.2, in some local coordinates the double point set of $g_{t}$ near the self-tangency point is given as follows:

$\left\{\left(x_{1}, \ldots, x_{2 k}, \mathbf{0}^{2 k-1}\right) \in \mathbb{R}^{4 k-1} \mid x_{1}^{2}+\cdots+x_{\lambda}^{2}-x_{\lambda+1}^{2}-\cdots-x_{2 k}^{2}=t\right\}$ in the $\boldsymbol{x}$-sheet, $\left\{\left(y_{1}, \ldots, y_{2 k}, \mathbf{0}^{2 k-1}\right) \in \mathbb{R}^{4 k-1} \mid y_{1}^{2}+\cdots+y_{\lambda}^{2}-y_{\lambda+1}^{2}-\cdots-y_{2 k}^{2}=t\right\} \quad$ in the $\boldsymbol{y}$-sheet.

The versal deformation transforms the double point set by a surgery that replaces $S^{\lambda-1} \times D^{2 k-\lambda}$ with $D^{\lambda} \times S^{2 k-(\lambda+1)}$ (see Figure 6.1). If $1<\lambda<2 k-1$, then this surgery transforms a single component in a small neighborhood of the self-tangency point and changes no linking numbers with other components. Thus $E\left(g_{t}\right)=E\left(g_{-t}\right)$.

If $\lambda=1$, then in each sheet the double point set has two components when $t>0$ : 


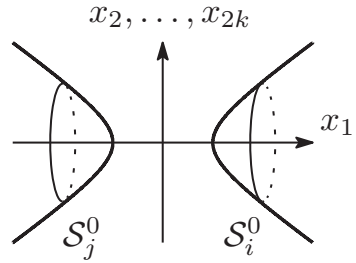

$t>0$

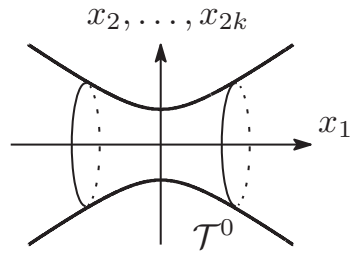

$t<0$

Figure 6.1: The double point set near a self-tangency point of index 1

in the $\boldsymbol{x}$-sheet

$$
\mathcal{S}_{i}^{0}:=\left\{x_{1}=\left(x_{2}^{2}+\cdots+x_{2 k}^{2}+t\right)^{1 / 2}\right\} \text { and } \mathcal{S}_{j}^{0}:=\left\{x_{1}=-\left(x_{2}^{2}+\cdots+x_{2 k}^{2}+t\right)^{1 / 2}\right\},
$$

and in the $\boldsymbol{y}$-sheet

$$
\mathcal{S}_{i}^{1}:=\left\{y_{1}=\left(y_{2}^{2}+\cdots+y_{2 k}^{2}+t\right)^{1 / 2}\right\} \text { and } \mathcal{S}_{j}^{1}:=\left\{y_{1}=-\left(y_{2}^{2}+\cdots+y_{2 k}^{2}+t\right)^{1 / 2}\right\}
$$

(Figure 6.1 , left). Here we choose $f_{t}$ so that it maps the $\boldsymbol{x}$-sheet to "below" the $\boldsymbol{y}$-sheet. In the $\boldsymbol{x}$-sheet, when $t<0, \mathcal{S}_{i}^{0} \sqcup \mathcal{S}_{j}^{0}$ is joined into a single component

$$
\mathcal{T}^{0}:=\left\{x_{1}^{2}-t=x_{2}^{2}+\cdots+x_{2 k}^{2}\right\}
$$

(Figure 6.1, right). Similarly, in the $\boldsymbol{y}$-sheet $\mathcal{S}_{i}^{1} \sqcup \mathcal{S}_{j}^{1}$ is joined into a single component $\mathcal{T}^{1}:=\left\{y_{1}^{2}-t=y_{2}^{2}+\cdots+y_{2 k}^{2}\right\}$ when $t<0$.

Let $L_{*}^{\epsilon}(*=i, j ; \epsilon=0,1)$ be the components of the double point set that contains $\mathcal{S}_{*}^{\epsilon}$ when $t>0$, and let $K^{\epsilon}$ be the component of the double point set containing $\mathcal{T}^{\epsilon}$ when $t<0$.

Case 1. Consider the case $A_{i} \neq A_{j}$. Here the number of the components of the self-intersection decreases by 1 when $t$ changes from $t>0$ to $-t$.

In this case, two components $L_{i}^{\epsilon} \sqcup L_{j}^{\epsilon}$ of the double point set of $g_{t}, t>0$, are joined into a connected double point set $K^{\epsilon}=L_{i}^{\epsilon} \sharp L_{j}^{\epsilon}$ of $g_{-t}(\epsilon=0,1)$. Other components $L_{m}^{\epsilon}(m \neq i, j ; \epsilon=0,1)$ are unchanged. The jump of the sum of linking numbers is thus

$$
\begin{gathered}
\sum_{(p, \epsilon)<\left(q, \epsilon^{\prime}\right)}(-1)^{\epsilon+\epsilon^{\prime}} l k\left(L_{p}^{\epsilon}\left(g_{t}\right), L_{q}^{\epsilon^{\prime}}\left(g_{t}\right)\right)-\sum_{(p, \epsilon)<\left(q, \epsilon^{\prime}\right)}(-1)^{\epsilon+\epsilon^{\prime}} l k\left(L_{p}^{\epsilon}\left(g_{-t}\right), L_{q}^{\epsilon^{\prime}}\left(g_{-t}\right)\right) \\
=\left(l k\left(L_{i}^{0}, L_{j}^{0}\right)+l k\left(L_{i}^{1}, L_{j}^{1}\right)-\sum_{p, q=i, j} l k\left(L_{p}^{0}, L_{q}^{1}\right)+\sum_{\substack{p=i, j ; m \neq i, j, \epsilon, \epsilon^{\prime}=0,1}}(-1)^{\epsilon+\epsilon^{\prime}} l k\left(L_{p}^{\epsilon}, L_{m}^{\epsilon^{\prime}}\right)\right) \\
-\left(-l k\left(K^{0}, K^{1}\right)+\sum_{m \neq i, j ; \epsilon, \epsilon^{\prime}=0,1}(-1)^{\epsilon+\epsilon^{\prime}} l k\left(K^{\epsilon}, L_{m}^{\epsilon^{\prime}}\right)\right) .
\end{gathered}
$$

The connected-sums $K^{\epsilon}=L_{i}^{\epsilon} \sharp L_{j}^{\epsilon}$ are taken near the tangency point and by a small isotopy we may assume that $L_{*}^{\epsilon}(*=i, j ; \epsilon=0,1)$ satisfy the condition in Lemma 5.1. Thus by Lemma 5.1

$$
\sum_{p, q=i, j} l k\left(L_{p}^{0}, L_{q}^{1}\right)=l k\left(K^{0}, K^{1}\right), \quad l k\left(L_{i}^{\epsilon}, L_{m}^{\epsilon^{\prime}}\right)+l k\left(L_{j}^{\epsilon}, L_{m}^{\epsilon^{\prime}}\right)=l k\left(K^{\epsilon}, L_{m}^{\epsilon^{\prime}}\right) .
$$


By the above three equations, we have

$$
E\left(g_{t}\right)-E\left(g_{-t}\right)= \pm \frac{l k\left(L_{i}^{0}, L_{j}^{0}\right)+l k\left(L_{i}^{1}, L_{j}^{1}\right)}{4} .
$$

Case 2. Consider the case when the versal deformation does not change the number of the components of the self-intersection-namely, $k>1$ and $A_{i}=A_{j}$. Since $L_{i}^{\epsilon}=L_{j}^{\epsilon}$ turns into $K^{\epsilon}$ and other components $L_{m}^{\epsilon}(m \neq i ; \epsilon=0,1)$ are unchanged, $E\left(g_{t}\right)=$ $E\left(g_{-t}\right)$ follows from the same argument as in the case $1<\lambda<2 k-1$.

Case 3. When $k=1$ and a negative self-tangency occurs at $t=0$ in $A_{i}=A_{j}$ (namely, two arcs get tangent to each other with opposite velocity vectors; by Lemma 2.3 no positive self-tangency occurs), the number of the components of the self-intersection increases by 1 when $t$ changes from $t>0$ to $-t$. This case is similar to Case 1.

The case $\lambda=2 k-1$ is similar, and we have the following.

Lemma 6.5. Suppose that $g_{0}$ has an indefinite self-tangency point. If the index of $Q$ is 1 or $2 k-1$, and if the versal deformation changes the number of the components of self-intersection, then

$$
E\left(g_{t}\right)-E\left(g_{-t}\right)= \pm \frac{l k\left(L_{i}^{0}, L_{j}^{0}\right)+l k\left(L_{i}^{1}, L_{j}^{1}\right)}{4},
$$

where $L_{i}^{0} \sqcup L_{i}^{1}=g_{t}^{-1}\left(A_{i}\right)$ and $L_{j}^{0} \sqcup L_{j}^{1}=g_{t}^{-1}\left(A_{j}\right)$ are the double point set corresponding to $A_{i}$ and $A_{j}$, the distinct components of the self-intersection of $g_{t}$ that are joined into a single component after the versal deformation. Otherwise, $E\left(g_{t}\right)=E\left(g_{-t}\right)$.

\subsubsection{Triple points}

Suppose $g_{0}$ is liftable and has a triple point. By Proposition 6.3, in some local coordinates, the double point set near the triple point in the $\boldsymbol{x}$-sheet is given by

$$
\begin{aligned}
& \mathcal{S}_{i}^{1}:=\left\{\left(x_{1}, \ldots, x_{2 k-1}, 0,0, \quad \ldots, 0\right)\right\}=+\mathbb{R}^{2 k-1} \times\{\mathbf{0}\}^{2 k}, \\
& \mathcal{S}_{j}^{1}:=\left\{\left(0, \ldots, 0, \quad t, x_{2 k+1}, \ldots, x_{4 k-1}\right)\right\}=\{\mathbf{0}\}^{2 k-1} \times\{t\} \times\left(+\mathbb{R}^{2 k-1}\right)
\end{aligned}
$$

as oriented manifolds (see Figure 6.2). The orientations are direct consequences of

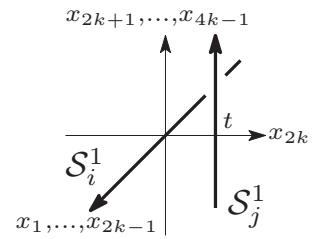

highest

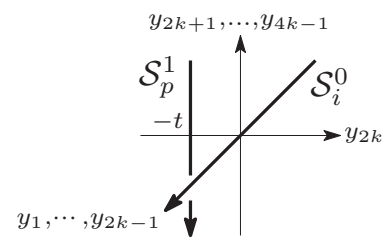

middle

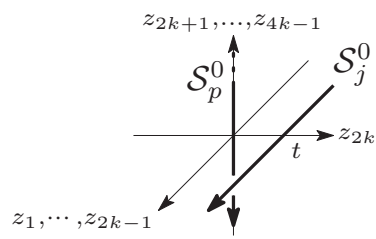

lowest

Figure 6.2: Double point sets near the triple point

Lemma 2.3. Similarly, the double point set in the $\boldsymbol{y}$-sheet is given by

$$
\begin{aligned}
& \mathcal{S}_{p}^{1}:=\left\{\left(0, \ldots, 0, \quad-t, y_{2 k+1}, \ldots, y_{4 k-1}\right)\right\}=\{\mathbf{0}\}^{2 k-1} \times\{-t\} \times\left(-\mathbb{R}^{2 k-1}\right), \\
& \mathcal{S}_{i}^{0}:=\left\{\left(y_{1}, \ldots, y_{2 k-1}, 0, \quad 0, \quad \ldots, 0\right)\right\}=+\mathbb{R}^{2 k-1} \times\{\mathbf{0}\}^{2 k},
\end{aligned}
$$


and in the $\boldsymbol{z}$-sheet by

$$
\begin{aligned}
& \mathcal{S}_{j}^{0}:=\left\{\left(z_{1}, \ldots, z_{2 k-1}, t, 0, \quad \ldots, 0\right)\right\}=+\mathbb{R}^{2 k-1} \times\{t\} \times\{\mathbf{0}\}^{2 k-1}, \\
& \mathcal{S}_{p}^{0}:=\left\{\left(0, \ldots, 0, \quad 0, z_{2 k+1}, \ldots, x_{4 k-1}\right)\right\}=\{\mathbf{0}\}^{2 k} \times\left(-\mathbb{R}^{2 k-1}\right) .
\end{aligned}
$$

Here, without loss of generality, we assume that the lift $f_{t}$ of $g_{t}$ maps the $\boldsymbol{x}$-sheet (in Proposition 6.3) to the "highest position," the $\boldsymbol{y}$-sheet to the "middle," and the $z$-sheet to the "lowest." The following holds by the above descriptions.

Lemma 6.6 (see [10, Remark 6.2.3]). The versal deformation changes three crossing $\mathcal{S}_{i}^{1} \sqcup \mathcal{S}_{j}^{1}, \mathcal{S}_{i}^{0} \sqcup \mathcal{S}_{p}^{1}$, and $\mathcal{S}_{j}^{0} \sqcup \mathcal{S}_{p}^{0}$, changing their linking numbers or writhes by the common value \pm 1 (the signs are same for all the three crossings).

Let $L_{*}^{\epsilon}(* \in\{i, j, p\}, \epsilon \in\{0,1\})$ be the component of the double point set containing $\mathcal{S}_{*}^{\epsilon}$.

Case 1. If all the six components $L_{*}^{\epsilon}$ are different, then by Lemma 6.6 the versal deformation changes $(-1)^{1+1} l k\left(L_{i}^{1}, L_{j}^{1}\right)+(-1)^{0+1} l k\left(L_{i}^{0}, L_{p}^{1}\right)+(-1)^{0+0} l k\left(L_{j}^{0}, L_{p}^{0}\right)$ by $\pm(1-1+1)= \pm 1$. Other linking numbers do not change. Thus $E\left(g_{t}\right)-E\left(g_{-t}\right)=$ $\pm 1 / 4$.

Case 2. If $L_{i}^{\epsilon}=L_{j}^{\epsilon} \neq L_{p}^{\epsilon}(\epsilon=0,1)$, then the crossing change at $\mathcal{S}_{i}^{0} \sqcup \mathcal{S}_{p}^{1}$ and $\mathcal{S}_{j}^{0} \sqcup \mathcal{S}_{p}^{0}$ changes $(-1)^{0+1} l k\left(L_{i}^{0}, L_{p}^{1}\right)+(-1)^{0+0} l k\left(L_{i}^{0}, L_{p}^{0}\right)$ by $\pm 1 \mp 1=0$. Other linking numbers do not change, and the change of $w\left(L_{i}^{1}\right)$ (by \pm 1 ) arising from the crossing change at $\mathcal{S}_{i}^{1} \sqcup \mathcal{S}_{j}^{1}$ does not change $E$. Thus $E\left(g_{t}\right)=E\left(g_{-t}\right)$.

Case 3. If $L_{p}^{\epsilon}=L_{i}^{\epsilon} \neq L_{j}^{\epsilon}(\epsilon=0,1)$, then the versal deformation changes $l k\left(L_{i}^{1}, L_{j}^{1}\right)-$ $l k\left(L_{i}^{1}, L_{i}^{0}\right)+l k\left(L_{i}^{0}, L_{j}^{0}\right)$ by $\pm(1-1+1)= \pm 1$, and $E\left(g_{t}\right)-E\left(g_{-t}\right)= \pm 1 / 4$.

Case 4 . The case $L_{j}^{\epsilon}=L_{p}^{\epsilon} \neq L_{i}^{\epsilon}$ is similar to the Case 2 by symmetry, and $E\left(g_{t}\right)=$ $E\left(g_{-t}\right)$.

Case 5. If $L_{i}^{\epsilon}=L_{j}^{\epsilon}=L_{p}^{\epsilon}$, then the versal deformation changes $-l k\left(L_{i}^{0}, L_{p}^{1}\right)$ by \pm 1 , and the changes of $w\left(L_{i}^{1}\right), w\left(L_{j}^{0}\right)$ do not affect $E$. Thus in this case $E\left(g_{t}\right)-E\left(g_{-t}\right)=$ $\pm 1 / 4$.

Putting them all together, we obtain the following.

Lemma 6.7. Suppose that $g_{0}$ has a triple point. Then $E\left(g_{t}\right)=E\left(g_{-t}\right)$ if $L_{i}^{\epsilon}=L_{j}^{\epsilon} \neq$ $L_{p}^{\epsilon}$ or $L_{j}^{\epsilon}=L_{p}^{\epsilon} \neq L_{i}^{\epsilon}, \epsilon=0,1$ (in Figure 6.2). Otherwise, $E\left(g_{t}\right)-E\left(g_{-t}\right)= \pm 1 / 4$.

6.4. The case $k=1$

For $g \in \mathcal{K}_{5,3}$ the invariant $E$ is essentially the Smale invariant;

Proposition 6.8 ([10, 30]). If $g \in \mathcal{K}_{5,3}$ (also regarded as $g \in \mathcal{K}_{6,3}$ by composing $\left.\mathbb{R}^{5} \hookrightarrow \mathbb{R}^{6}\right)$, then $\mathcal{H}(g)=E(g)=-\Omega(g) / 12$, where $\Omega: \pi_{0}\left(\mathcal{I}_{5,3}\right) \stackrel{\cong}{\rightrightarrows} \mathbb{Z}$ is the Smale invariant.

Proof. $\mathcal{H}=E$ follows from (2.4), since $g$ has no self-intersection. As explained in [10], there exists a "Seifert surface" for $g$-that is, an embedding $V^{4} \hookrightarrow \mathbb{R}^{5}$ that restricts to $g: \partial V=\mathbb{R}^{3} \hookrightarrow \mathbb{R}^{5}$ - and $\Omega(g)=3 \sigma(V) / 2$, where $\sigma$ denotes the signature. [30, Corollary 2.4] states that $\mathcal{H}(g)=-\sigma(V) / 8\left(e_{F}=0\right.$ for $\left.g \in \mathcal{K}_{5,3}\right)$.

Remark 6.9. If $g \in \mathcal{K}_{5,3}$, then $E(g)=I(Y)(g) / 6+c(g)$ by (6.1). Thus $\Omega(g)=$ $-2 I(Y)(g)-12 c(g)$. 
The double point set of generic $g \in \mathcal{I}_{5,3}$ is a classical link. A result of Ogasa [21] characterizes which link can be realized as a double point set of an immersion $\mathbb{R}^{3} \rightarrow \mathbb{R}^{5}$.

Theorem 6.10 (A special case of [21, Theorem 1.1]). For any link $L \subset S^{3}$, there exist embeddings $g^{\epsilon}: S^{3} \hookrightarrow \mathbb{R}^{5}(\epsilon=0,1)$ such that $\left(g^{\epsilon}\right)^{-1}\left(g^{0}\left(S^{3}\right) \cap g^{1}\left(S^{3}\right)\right)(\epsilon=0,1)$ are isotopic to the given $L$. Moreover, we can choose $g^{\epsilon}$ to be isotopic to the natural inclusion.

Using this, we show the second half of Theorem 2.11 and Corollary 2.7.

Proof of Theorem 2.11, the second half. We show that an arbitrarily large jump of $E$ at a single indefinite self-tangency can occur. Any linear combination of Ekholm invariants $J$ and $S t$ cannot satisfy this property, because their jumps are bounded [10].

For any two-component link $L=K_{1} \cup K_{2}$ in $S^{3}$, choose $g^{\epsilon}: S^{3} \hookrightarrow \mathbb{R}^{5}, \epsilon=0,1$ as in Theorem 6.10. Taking a suitable connected-sum of the standard inclusion $f_{0}: \mathbb{R}^{3} \hookrightarrow \mathbb{R}^{5}$ with $g^{0}$ and $g^{1}$, we obtain $g:=f_{0} \sharp g^{0} \sharp g^{1} \in \mathcal{I}_{5,3}$, which satisfies the following conditions:

(i) The self-intersection $A=A_{1} \sqcup A_{2}$ of $g$ satisfies $g^{-1}\left(A_{i}\right)=K_{i}^{0} \cup K_{i}^{1}$ with each $K_{1}^{\epsilon} \cup K_{2}^{\epsilon}$ included in the $g^{\epsilon}$-part $\left(g^{\epsilon}\right)^{-1}(A)$ and isotopic to the given link $L$.

(ii) A lift of $g$ exists and can be obtained by lifting $g^{1}$-part into $\mathbb{R}^{5} \times \mathbb{R}_{+}$and letting $g^{0}$-part remain inside $\mathbb{R}^{5} \times\{0\}$.

$K_{1}^{0} \cup K_{2}^{0}$ is separated from $K_{1}^{1} \cup K_{2}^{1}$ by (i), above. Take $q_{i} \in A_{i}$ and $p_{i}^{\epsilon} \in K_{i}^{\epsilon}$ so that $g^{\epsilon}\left(p_{i}^{\epsilon}\right)=q_{i}$. Choose paths $\gamma^{\epsilon}:[0,1] \rightarrow \mathbb{R}^{3} \backslash\left(g^{\epsilon}\right)^{-1}(A)$ from $p_{1}^{\epsilon}$ to $p_{2}^{\epsilon}$ in the $g^{\epsilon}$-part. Then $C:=g\left(\gamma^{0}([0,1]) \cup \gamma^{1}([0,1])\right)$ is a circle in $\mathbb{R}^{5}$, which can be seen as the image of a trivial knot. Thus $C$ bounds an embedded 2-disk $D$ in $\mathbb{R}^{5}$ whose interior transversely intersects $g$ at finitely many points outside $K_{i}^{\epsilon}$ (Figure 6.3). There is a homotopy that transforms $g$ only near $\gamma^{0}([0,1])$ so that $g\left(\gamma^{0}([0,1])\right)$ gets close to $g\left(\gamma^{1}([0,1])\right)$ along $D$ and eventually $D$ does not intersect $g$ in its interior. During this homotopy a number of non-generic self-intersections may appear, but $A_{1} \sqcup A_{2}$ remains unchanged. Thus we get $g^{\prime} \in \mathcal{I}_{5,3}, g^{\prime} \simeq g$, for which Int $D \cap g^{\prime}=\emptyset$ and we can choose a local coordinate of $\mathbb{R}^{5}$ around the tubular neighborhood of $D$ so that

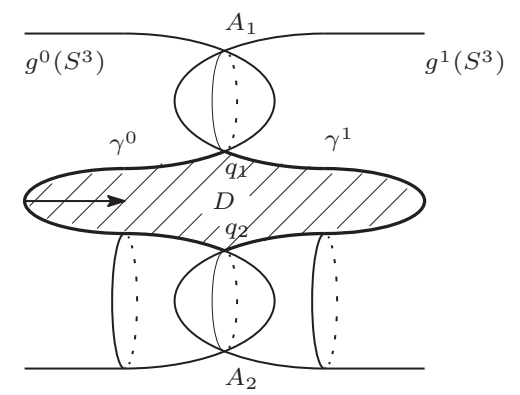

Figure 6.3: The self-intersection of $g$ in the proof of Theorem 2.11

(1) a tubular neighborhood of $g^{\prime}\left(\gamma^{0}([0,1])\right)$ in $\mathbb{R}^{3}$ corresponds to $\left\{\left(x_{1}, x_{2}, x_{3}, 0,0\right) \mid\right.$ $\left.x_{1}^{2}+x_{3}^{2}<r,\left|x_{2}\right| \leqslant 1\right\} \quad(r>0$ small $)$,

(2) a tubular neighborhood of $g^{\prime}\left(\gamma^{1}([0,1])\right)$ in $\mathbb{R}^{3}$ corresponds to $\left\{\left(x_{1}, x_{2}, 0, x_{1}^{2}-\right.\right.$ $\left.\left.x_{2}^{2}+1, x_{5}\right)\left|x_{1}^{2}+x_{5}^{2}<r,\right| x_{2} \mid \leqslant 1\right\}$, and 
(3) $D$ corresponds to $\left\{\left(0, x_{2}, 0, x_{4}, 0\right) \mid 0 \leqslant x_{4} \leqslant 1-x_{2}^{2}\right\}$

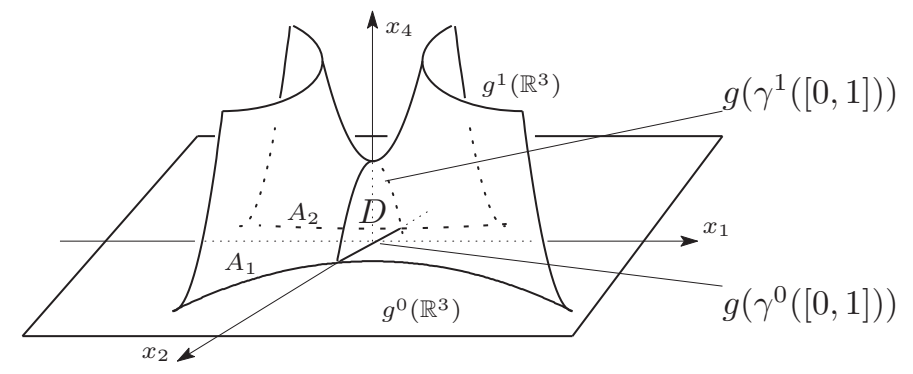

Figure 6.4: A neighborhood of $D$ in $x_{1} x_{2} x_{4}$-plane

(see Figure 6.4). This coordinate coincides with that in Proposition 6.2, and there exists a regular homotopy of $g^{\prime}$ inside this coordinate in which an indefinite selftangency between $A_{1}$ and $A_{2}$ occurs. By Lemma $6.5, E$ jumps by $\pm\left(l k\left(K_{1}^{0}, K_{2}^{0}\right)+\right.$ $\left.l k\left(K_{1}^{1}, K_{2}^{1}\right)\right) / 4= \pm l k\left(K_{1}, K_{2}\right) / 2$ at this self-tangency. This jump can be arbitrarily large, since $L=K_{1} \sqcup K_{2}$ is arbitrary.

Proof of Corollary 2.7. The immersion $g$ constructed in the proof of Theorem 2.11 can be lifted to $f \in \mathcal{K}_{6,3}$ by lifting the $g^{1}$-part into $\mathbb{R}^{5} \times \mathbb{R}_{+}$. Since $g^{\epsilon}$ 's are isotopic to the standard embedding, we see that $\mathcal{H}(f)=0$. Changing the crossing at $A_{1}$, by (2.2) we have

$$
\begin{aligned}
0-\mathcal{H}\left(f_{\{1\}}\right) & =\frac{1}{2}\left(l k\left(K_{1}^{0}, K_{2}^{0}\right)-l k\left(K_{1}^{0}, K_{2}^{1}\right)-l k\left(K_{1}^{1}, K_{2}^{0}\right)+l k\left(K_{1}^{1}, K_{2}^{1}\right)\right) \\
& =l k\left(K_{1}, K_{2}\right) .
\end{aligned}
$$

Here we use the fact that $K_{i}^{0}$ and $K_{j}^{1}$ are separated and that $K_{1}^{\epsilon} \sqcup K_{2}^{\epsilon}$ is isotopic to the given link $L=K_{1} \sqcup K_{2}$. This means that an embedding $\mathbb{R}^{3} \hookrightarrow \mathbb{R}^{6}$ with arbitrary Haefliger invariant can be obtained by a single crossing change from the trivial embedding.

\section{Appendix A. The Jacobian in the proof of Proposition 5.3}

\begin{tabular}{|c|c|c|c|c|c|c|c|c|c|}
\hline$I_{2 k-1}$ & & $-I_{2 k-1}$ & & & & & & & \\
\hline & $I_{2 k}$ & & & & & & & & \\
\hline & & & $-I_{2 k}$ & & & & & & \\
\hline & & & & & & & $-I_{2 k-1}$ & & \\
\hline & & & & $I_{2 k-1}$ & & & & & \\
\hline & & & & & $I_{2 k-1}$ & 0 & & $-I_{2 k-1}$ & 0 \\
\hline & & & & & ${ }^{t} \mathbf{0}$ & 1 & & ${ }^{t} \mathbf{0}$ & 0 \\
\hline & & & & & ${ }^{t} \mathbf{0}$ & 0 & & ${ }^{t} 0$ & -1 \\
\hline & & $I_{2 k-1}$ & & $-I_{2 k-1}$ & & & & & \\
\hline & & & $I_{2 k-1} \mid \mathbf{0}$ & & $-I_{2 k-1}$ & 0 & & & \\
\hline
\end{tabular}

The Jacobian matrix $J\left(\varphi_{X}\right)$ at $\vec{\xi}$ in the proof of Proposition 5.3 is given by 
where $I_{N}$ is the $(N \times N)$-identity matrix and $\mathbf{0} \in \mathbb{R}^{2 k-1}$ is the zero vector. The rows correspond to the bases of $T_{\left(e_{6 k-1}, e_{6 k-1}, e_{4 k-1}\right)}\left(S^{6 k-1} \times S^{6 k-1} \times S^{4 k-2}\right)$ and the

columns correspond to the natural basis of $T_{\vec{\xi}} \operatorname{Conf}_{4}\left(\mathbb{R}^{4 k-1}\right) \cong T_{\vec{\xi}} \mathbb{R}^{16 k-4}$. Its determinant is -1 .

\section{References}

[1] V. Arnold, Plane curves, their invariants, perestroikas and classifications, Singularities and bifurcations, Adv. Soviet Math., vol. 21, Amer. Math. Soc., Providence, RI, 1994, pp. 33-91.

[2] G. Arone and V. Turchin, On the rational homology of high-dimensional analogues of spaces of long knots, Geom. Topol. 18 (2014), no. 3, 1261-1322.

[3] J. Birman and X.-S. Lin, Knot polynomials and Vassiliev's invariants, Invent. Math. 111 (1993), no. 2, 225-270.

[4] R. Bott and C. Taubes, On the self-linking of knots, J. Math. Phys. 35 (1994), no. $10,5247-5287$.

[5] R. Budney, A family of embedding spaces, Groups, homotopy and configuration spaces (Tokyo 2005), Geom. Topol. Monogr., vol. 13, 2008, pp. 41-84.

[6] R. Budney, J. Conant, K. P. Scannell, and D. Sinha, New perspectives on selflinking, Adv. in Math. 191 (2005), no. 1, 78-113.

[7] R. Budney, J. Conant, R. Koytcheff, and D. Sinha, Embedding calculus knot invariants are of finite type, arXiv:1411.1832.

[8] A. Cattaneo, P. Cotta-Ramusino, and R. Longoni, Configuration spaces and Vassiliev classes in any dimensions, Algebr. Geom. Topol. 2 (2002), 949-1000.

[9] A. Cattaneo and C. Rossi, Wilson surfaces and higher dimensional knot invariants, Comm. Math. Phys. 256 (2005), no. 3, 513-537.

[10] T. Ekholm, Differential 3-knots in 5-space with and without self-intersection, Topology 40 (2001), 157-196.

[11] T. Ekholm, Invariants of generic immersions, Pac. J. Math. 199 (2001), no. 2, 321-345.

[12] T. Goodwillie and M. Weiss, Embeddings from the point of view of immersion theory II, Geom. Topol. 3 (1999), 103-118.

[13] K. Habiro, T. Kanenobu, and A. Shima, Finite type invariants of ribbon 2knots, Low-dimensional topology (Funchal, 1998), 187-196, Contemp. Math., 233, Amer. Math. Soc., Providence, RI, 1999.

[14] A. Haefliger, Knotted $(4 k-1)$-spheres in 6k-space, Ann. of Math. (2) 75 (1962), $452-466$.

[15] T. Kohno, Vassiliev invariants and de Rham complex on the space of knots, Contemp. Math., 179 (1994), 123-138.

[16] X.-S. Lin and Z. Wang, Integral geometry of plane curves and knot invariants, J. Differential Geom. 44 (1996), no. 1, 74-95.

[17] R. Milgram, On the Haefliger knot groups, Bull. Amer. Math. Soc. 78 (1972), 861-865. 
[18] B. Munson, Embeddings in the 3/4 range, Topology 44 (2005), no. 6, 11331157.

[19] T. Ochiai, The combinatorial Gauss diagram formula for Kontsevich integral, J. Knot Theory Ramifications 10 (2001), no. 6, 851-906.

[20] T. Ochiai, Invariants of plane curves and Polyak-Viro type formulas for Vassiliev invariants, J. Math. Sci. Univ. Tokyo 11 (2004), no. 2, 155-175.

[21] E. Ogasa, The intersection of spheres in a sphere and a new geometric meaning of the Arf invariant, J. Knot Theory Ramifications 11 (2002), no. 8, 1211-1231.

[22] K. Ohba, The unknotting numbers of knotted 3-spheres in the 6-sphere in the sense of Haefliger, RIMS kokyuroku 1393 (2004), 132-139 (written in Japanese).

[23] M. Polyak and O. Viro, On the Casson knot invariant, J. Knot Theory Ramifications 10 (2001), no. 5, 711-738.

[24] D. Roseman and M. Takase, High-codimensional knots spun about manifolds, Algebr. Geom. Topol. 7 (2007), 359-377.

[25] K. Sakai, Configuration space integral for embedding spaces and the Haefliger invariant, J. Knot Theory Ramifications 19 (2010), no. 12, 1597-1644.

[26] K. Sakai and T. Watanabe, 1-loop graphs and configuration space integral for embedding spaces, Math. Proc. Cambridge Philos. Soc. 152 (2012), no. 3, 497533.

[27] D. Sinha, Manifold theoretic compactifications of configuration spaces, Selecta Math. (new ser.) 10 (2004), no. 3, 391-428.

[28] M. Takase, A geometric formula for Haefliger knots, Topology 43 (2004), no. 6, $1425-1447$.

[29] M. Takase, Homology 3-spheres in codimension three, Internat. J. Math. 17 (2006), no. 8, 869-885.

[30] M. Takase, The Hopf invariant of a Haefliger knot, Math. Z. 256 (2007), no. 1, $35-44$.

[31] I. Volić, Finite type knot invariants and calculus of functors, Compos. Math. 142 (2006), no. 1, 222-250.

[32] I. Volić, Configuration space integrals and Taylor towers for spaces of knots, Topology Appl. 153 (2006), no. 15, 2893-2904.

[33] T. Watanabe, Configuration space integral for long n-knots and the Alexander polynomial, Algebr. Geom. Topol. 7 (2007), 47-92.

Keiichi Sakai ksakai@math.shinshu-u.ac.jp

Faculty of Science, Shinshu University, 3-1-1 Asahi, Matsumoto, Nagano 390-8621, Japan 